\title{
Common origin of baryon asymmetry, Dark Matter and neutrino mass
}

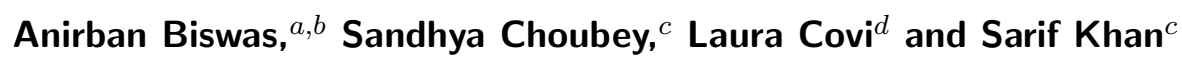 \\ ${ }^{a}$ Department of Physics, Indian Institute of Technology Guwahati, \\ Assam 781039, India \\ ${ }^{b}$ School of Physical Sciences, Indian Association for the Cultivation of Science, \\ 2A 83 2B Raja S.C. Mullick Road, Kolkata 700032, India \\ ${ }^{c}$ Harish-Chandra Research Institute, HBNI, \\ Chhatnag Road, Jhunsi, Allahabad 211 019, India \\ ${ }^{d}$ Institute for Theoretical Physics, Georg-August University Göttingen, \\ Friedrich-Hund-Platz 1, Göttingen D-3707\%, Germany \\ E-mail: anirban.biswas.sinp@gmail.com, sandhya@hri.res.in, \\ laura.covi@theorie.physik.uni-goettingen.de, sarifkhan@hri.res.in
}

ABSTRACT: In this work, we explain three beyond standard model (BSM) phenomena, namely neutrino masses, the baryon asymmetry of the Universe and Dark Matter, within a single model and in each explanation the right handed $(\mathrm{RH})$ neutrinos play the prime role. Indeed by just introducing two $\mathrm{RH}$ neutrinos we can generate the neutrino masses by the Type-I seesaw mechanism. The baryon asymmetry of the Universe can arise from thermal leptogenesis from the decay of lightest $\mathrm{RH}$ neutrino before the decoupling of the electroweak sphaleron transitions, which redistribute the $B-L$ number into a baryon number. At the same time, the decay of the $\mathrm{RH}$ neutrino can produce the Dark Matter $(\mathrm{DM})$ as an asymmetric Dark Matter component. The source of $\mathrm{CP}$ violation in the two sectors is exactly the same, related to the complex couplings of the neutrinos. By determining the comoving number density for different values of the $\mathrm{CP}$ violation in the DM sector, we obtain a particular value of the DM mass after satisfying the relic density bound. We also give prediction for the DM direct detection (DD) in the near future by different ongoing DD experiments.

KeYwords: Cosmology of Theories beyond the SM, Neutrino Physics

ARXIV EPRINT: 1812.06122 


\section{Contents}

1 Introduction 1

2 Model 3

2.1 Particle spectrum 3

2.2 Lagrangian 3

$\begin{array}{lll}3 & \text { Neutrino mass } & 6\end{array}$

4 Boltzmann equation for studying lepton asymmetry and Dark Matter asymmetry

$\begin{array}{llr}5 & \text { Results } & 12\end{array}$

6 Direct detection of Dark Matter $\quad 17$

$\begin{array}{llr}7 \text { Conclusion } & 18\end{array}$

$\begin{array}{ll}\text { A Expression for the Majorana mass matrix of light neutrinos } & 21\end{array}$

B Expression of $\gamma_{D_{1}}, \gamma_{\phi_{h}, s}^{1}$ and $\gamma_{\phi_{h}, t}^{1}: \quad 22$

\section{Introduction}

The Standard model (SM) is a concrete and successful theory which describes beautifully all the past and present particle physics measurements at colliders and at low energy experiments. After the recent discovery of the Higgs boson, all the particles of the SM have been detected and are fully consistent so far with the SM predictions. In spite of this terrific success, we know though that we still do not have a complete picture of particle physics. Indeed on one side we have clear evidence for neutrino masses, by observing the neutrino oscillation among the different flavours in atmospheric, solar and reactor neutrinos [1-11]. On the other hand, we know from astrophysical and cosmological data that in the Universe we need an additional matter component, apart for baryonic matter, to make up around $26 \%$ of the total energy budget. There are many evidences which support the existence of DM, mainly flatness of the galaxy rotation curves, the observation of bullet cluster and the CMB anisotropy [12-18]. In order to satisfy the observations DM has to be electrically neutral and stable or the decay life time has to be much longer than the age of the Universe (e.g. see ten point test of becoming the DM candidate in [19]). No particle of the SM has the right characteristics to provide the main Dark Matter component. Indeed neutrinos, which are neutral and stable, are unfortunately too light and cannot be the sole Dark Matter particle, see e.g. [20]. 
Another big puzzle is that there exist an excess of baryonic matter over anti-baryonic matter in the Universe and the baryon asymmetry has been measured very precisely by the satellite-based experiments WMAP and Planck [16-18]. It turns out that the baryonic matter makes up approximately $5 \%$ of the present energy density of the Universe and is approximately a factor 5 less abundant than Dark Matter. In order to generate the baryon asymmetry of the Universe, we need to satisfy the Sakharov conditions [21] including sufficient $\mathrm{C}$ and $\mathrm{CP}$ violation and deviation from thermal equilibrium. These conditions are difficult to realize within the SM and require generically new physics.

In this work, we mainly address the above mentioned three puzzles and try to solve them in a unified manner through the presence of $\mathrm{RH}$ handed-neutrinos, which mediate with the Dark Matter sector. Indeed the introduction of at least two RH neutrinos, singlets under the SM gauge group, allows to generate Majorana masses for the light neutrinos by the Type-I seesaw mechanism [22, 23]. Moreover, it is well known that RH neutrinos can also generate the baryon asymmetry of the Universe via leptogenesis [24, 25]. The lepton number asymmetry generated in the lightest $\mathrm{RH}$ neutrino decay can in fact be partially converted into a baryon asymmetry by sphaleron processes, which remain in thermal equilibrium until the electroweak phase transition [26].

Regarding the Dark Matter, the most popular ways to generate the appropriate density, like the freeze-out or freeze-in mechanisms, are usually independent from the neutrino sector and from baryogenesis. But in our work, we will instead follow the paradigm of asymmetric Dark Matter (ADM) also in order to explain the comparable densities of baryons and DM. Indeed if the RH neutrinos not only have a Yukawa coupling with the light neutrinos and the SM Higgs, but also couple with the Dark Matter sector, they can decay also in the Dark Sector generating an asymmetry of a similar order. Many models of asymmetric Dark Matter have been proposed in the literature [27-52], but in this work we will give a new realization of the scenario and exploit and explore more in depth the connection to neutrino physics.

In order to solve the above mentioned problems in a common way, we extend the SM both in the particle content as well as in the gauge group structure. We add to the gauge group a local $\mathrm{SU}(2)_{D}$ interaction $^{1}$ in the Dark sector and discrete $\mathbb{Z}_{3}, \mathbb{Z}_{2}$ groups, ensuring the Dark Matter stability as well as forbidding the Majorana mass terms among the dark sector particles and limiting the number of model parameters. The particle list is also enlarged to include two dark sector fermionic left handed doublets and their $\mathrm{RH}$ counterparts, singlet under the dark $\mathrm{SU}(2)_{D}$, two scalar doublets of $\mathrm{SU}(2)_{D}$ and two singlet $\mathrm{RH}$ neutrinos. We must introduce two $\mathrm{SU}(2)_{D}$ fermionic doublets in order to cancel the Witten anomaly [54]. As we will see in the result section, the RH neutrinos take part in generating the lepton asymmetry, Dark Matter asymmetry and neutrino mass. One of the scalar doublets takes a vacuum expectation value, generating a mass for the exotic fermions and also mixing with the SM Higgs. This opens up the possibility to have DM scatterings mediated by the Higgs fields, which may be detected in different ongoing and proposed direct detection experiments [55-61]. The other exotic scalar doublet does not

\footnotetext{
${ }^{1}$ WIMP type DM obeying SU(2) gauge symmetry has been studied earlier in [53].
} 
obtain a non-zero vacuum expectation value and plays a role similar to the inert doublet, participating in our case to the DM production.

The paper is organised in the following way. In section 2, we describe all the details of our model. The generation of the neutrino mass is discussed in section 3. In section 4, we give the Boltzmann equations for both the dark sector and the leptonic sector, while section 5, contains the full numerical result of the Boltzmann equations. In section 6 , the DM direct detection is addressed. Finally, in section 7, we conclude our work with an outlook on possible signature at colliders.

\section{Model}

In this article, we consider a hidden sector which has a local $\mathrm{SU}(2)_{D}$ gauge invariance. In this hidden sector we introduce four fermions $\psi_{i}(i=1$ to 4$)$ whose left handed components transform like a doublet under $\mathrm{SU}(2)_{D}$ while the right handed counterparts are $\mathrm{SU}(2)_{D}$ singlets. Therefore, we have a pair of $\mathrm{SU}(2)_{D}$ doublets $\Psi_{\alpha L}(\alpha=1,2)$. In table 1 , we show the complete list of particles in the present model. Here we want to point that, since we have an even number of fermionic $\mathrm{SU}(2)_{D}$ doublets, our model is free from the Witten anomaly [54]. In addition, we have two scalar doublets in the hidden sector as well. One of the scalar doublets $\eta_{D}$ does not get any vacuum expectation value (VEV) while the remaining one $\left(\phi_{D}\right)$ has a nonzero VEV and thus mixes with the SM Higgs doublet $\phi_{h}$. Moreover, in order to have a stable DM candidate, we also impose a discrete $\mathbb{Z}_{3}$ symmetry, and keep all the hidden sector fermions as well as the inert doublet charged under $\mathbb{Z}_{3}$. These symmetries allow Majorana mass terms among the extra $\mathrm{SU}(2)_{D}$ singlet fermions, which after the breaking of the dark symmetry could switch on the conversion of the DM to anti-DM. Therefore, to be on the safe side we introduce an additional $\mathbb{Z}_{2}$ symmetry to forbid the Majorana mass terms among the extra fermions and reduce the possible couplings of the second fermionic state. Under $\mathbb{Z}_{2}, \psi_{2 L}, \psi_{3 R}$ and $\psi_{4 R}$ are odd and the rest of the particles including the Standard Model particles are even. Furthermore, we have two right handed (RH) fermions $N_{i}(i=1,2)$, singlets under both SM gauge group as well as $\mathrm{SU}(2)_{D}$. These singlet fermions play the role of the $\mathrm{RH}$ neutrino and are the only connector between the visible and the hidden sector, as long as the electroweak and dark $\mathrm{SU}(2)_{D}$ are unbroken and the mixing in the scalar sector vanishes. In this sense our model is a special case in the class of neutrino(+Higgs) portal models [62-67].

\subsection{Particle spectrum}

\subsection{Lagrangian}

The $\mathrm{SU}(3)_{c} \times \mathrm{SU}(2)_{L} \times \mathrm{SU}(2)_{D} \times \mathrm{U}(1)_{Y} \times \mathbb{Z}_{3}$ invariant Lagrangian for our present model is given by,

$$
\begin{aligned}
\mathcal{L}= & \mathcal{L}_{\mathrm{SM}}+i \overline{\Psi_{k}} \gamma^{\mu} D_{\mu}^{k} \Psi_{k}+\left(D_{\mu}^{D} \phi_{D}\right)^{\dagger}\left(D^{D \mu} \phi_{D}\right)+\left(D_{\mu}^{D} \eta_{D}\right)^{\dagger}\left(D^{D \mu} \eta_{D}\right)+\left(y_{i j} \bar{L}_{i} \tilde{\phi}_{h} N_{j R}+\text { h.c. }\right) \\
& +\left(\lambda_{1} \overline{\Psi_{1 L}} \tilde{\phi_{D}} \psi_{1 R}+\lambda_{2} \overline{\Psi_{1 L}} \phi_{D} \psi_{2 R}+\lambda_{3} \overline{\Psi_{2 L}} \tilde{\phi_{D}} \psi_{3 R}+\lambda_{4} \overline{\Psi_{2 L}} \phi_{D} \psi_{4 R}+\text { h.c. }\right) \\
& -\alpha_{j} \overline{\Psi_{1 L}} \eta_{D} N_{j_{R}}+i \overline{N_{j R}} \not \partial N_{j_{R}}-M_{j} \overline{N_{j}^{c}} N_{j_{R}}-\mathcal{V}\left(\phi_{h}, \phi_{D}, \eta_{D}\right),
\end{aligned}
$$




\begin{tabular}{|c|c|c|c|c|c|c|c|c|c|c|}
\hline \multirow{2}{*}{$\begin{array}{l}\text { Gauge } \\
\text { Group }\end{array}$} & \multicolumn{7}{|c|}{ Fermion Fields } & \multicolumn{3}{|c|}{ Scalar Fields } \\
\hline & $\Psi_{1 L}=\left(\psi_{1}, \psi_{2}\right)_{L}^{T}$ & $\psi_{1 R}$ & $\psi_{2 R}$ & $\Psi_{2 L}=\left(\psi_{3}, \psi_{4}\right)_{L}^{T}$ & $\psi_{3 R}$ & $\psi_{4 R}$ & $N_{i}$ & $\phi_{h}$ & $\phi_{D}$ & $\eta_{D}$ \\
\hline $\mathrm{SU}(3)_{c}$ & 1 & 1 & 1 & 1 & 1 & 1 & 1 & 1 & 1 & 1 \\
\hline $\mathrm{SU}(2)_{L}$ & 1 & 1 & 1 & 1 & 1 & 1 & 1 & 2 & 1 & 1 \\
\hline $\mathrm{SU}(2)_{D}$ & 2 & 1 & 1 & 2 & 1 & 1 & 1 & 1 & 2 & 2 \\
\hline $\mathbb{Z}_{3} \times \mathbb{Z}_{2}$ & $(\omega, 1)$ & $(\omega, 1)$ & $(\omega, 1)$ & $\left(\omega^{2},-1\right)$ & $\left(\omega^{2},-1\right)$ & $\left(\omega^{2},-1\right)$ & $(1,1)$ & $(1,1)$ & $(1,1)$ & $(\omega, 1)$ \\
\hline
\end{tabular}

Table 1. List of hidden sector particles and connector particles and their corresponding charges under various symmetry groups. All the particles listed above have zero hypercharge except SM Higgs doublet $\phi_{h}$ which has hypercharge $Y=1 / 2$.

where $i=1$ to 3 while $j$ runs from 1 to 2 and $D_{\mu}^{D}$ is the covariant derivative for $\mathrm{SU}(2)_{D}$. We assign the $\mathbb{Z}_{3}$ charges to the different fields in such a way so that the lightest component of the dark doublet $\Psi_{1 L}$ becomes stable and a viable DM candidate. Moreover, in the present work we are interested in $\psi_{1},{ }^{2}$ production from the decays of RH-neutrinos. Note that we can consider this state to have all real couplings by absorbing all the phases in $\lambda_{1,2}$, in the RH states $\psi_{1,2 R}$, while the phases of $\alpha_{1,2}$ can be absorbed into the doublets $\Psi_{1 L}$ and $\eta_{D}$. Without loss of generality we can also consider the heavy Majorana masses $M_{1,2}$ to be real and positive, but redefining accordingly the heavy $\mathrm{RH}$ neutrino fields $N_{1,2 R}$.

In the model there is also a second state, possibly $\psi_{3}$, which is stable due to the $Z_{2}$ and $Z_{3}$ symmetries and could contribute to the Dark Matter density. To avoid both a substantial $\psi_{3}$ freeze-out density and a symmetric DM component from $\psi_{1}$, the presence of the $\mathrm{SU}(2)_{D}$ gauge symmetry is crucial since it allows for an efficient annihilation of the fermions, as long as they are not too heavy. We will discuss the precise value of the allowed mass range later on. In this way, the main component of the DM in the Universe will be generated by the DM asymmetry in the RH neutrino's decays.

Given the symmetries discussed above, the gauge invariant scalar potential has the following form

$$
\begin{aligned}
\mathcal{V}\left(\phi_{h}, \phi_{D}, \eta_{D}\right)= & -\mu_{h}^{2}\left(\phi_{h}^{\dagger} \phi_{h}\right)+\lambda_{h}\left(\phi_{h}^{\dagger} \phi_{h}\right)^{2}-\mu_{D}^{2}\left(\phi_{D}^{\dagger} \phi_{D}\right)+\lambda_{D}\left(\phi_{D}^{\dagger} \phi_{D}\right)^{2} \\
& +\mu_{\eta}^{2}\left(\eta_{D}^{\dagger} \eta_{D}\right)+\lambda_{\eta}\left(\eta_{D}^{\dagger} \eta_{D}\right)^{2}+\lambda_{h D}\left(\phi_{h}^{\dagger} \phi_{h}\right)\left(\phi_{D}^{\dagger} \phi_{D}\right)+\lambda_{h \eta}\left(\phi_{h}^{\dagger} \phi_{h}\right)\left(\eta_{D}^{\dagger} \eta_{D}\right) \\
& +\lambda_{D 1}\left(\phi_{D}^{\dagger} \phi_{D}\right)\left(\eta_{D}^{\dagger} \eta_{D}\right)+\lambda_{D 2}\left(\phi_{D}^{\dagger} \eta_{D}\right)\left(\eta_{D}^{\dagger} \phi_{D}\right)+\lambda_{D 3}\left(\phi_{D} \eta_{D}^{3}+\text { h.c. }\right) .
\end{aligned}
$$

Here, both the doublets $\phi_{h}$ and $\phi_{D}$ acquire VEVs and generate masses to SM particles and the hidden sector fermions after spontaneous breaking of $\mathrm{SU}(2)_{L} \times \mathrm{U}(1)_{Y}$ and $\mathrm{SU}(2)_{D}$ symmetries, respectively. In the unitary gauge, scalar doublets $\phi_{h}$ and $\phi_{D}$ take the following form after symmetry breaking,

$$
\phi_{h}=\left(\begin{array}{c}
0 \\
\frac{v+h}{\sqrt{2}}
\end{array}\right), \quad \phi_{D}=\left(\begin{array}{c}
0 \\
\frac{v_{D}+H}{\sqrt{2}}
\end{array}\right) .
$$

\footnotetext{
${ }^{2}$ Like the SM lepton doublets, in dark sector too, we have assumed that between the components of a dark doublet the component with isospin $+1 / 2$ is the lightest one.
} 
In the scalar potential there is a mixing term between the CP even neutral components of the two doublets $\phi_{h}$ and $\phi_{D}$, hence the gauge basis and mass eigenbasis will be different. In $h, H$ basis the mass square mixing matrix will be as follows

$$
M_{\text {scalar }}^{2}=\left(\begin{array}{cc}
2 \lambda_{h} v^{2} & \lambda_{h D} v v_{D} \\
\lambda_{h D} v v_{D} & 2 \lambda_{D} v_{D}^{2}
\end{array}\right) .
$$

After diagonalising the above mass matrix we get the physical masses and the corresponding physical states which are linear combinations of gauge basis in the following manner

$$
\begin{aligned}
& h_{1}=h \cos \zeta-H \sin \zeta, \\
& h_{2}=h \sin \zeta+H \cos \zeta,
\end{aligned}
$$

where $\zeta$ is the mixing angle between $h_{1}, h_{2}$ and the mixing angle can be expressed in terms of the Lagrangian parameters in following way

$$
\tan 2 \zeta=\frac{\lambda_{h D} v v_{D}}{\lambda_{D} v_{D}^{2}-\lambda_{h} v^{2}} .
$$

As mentioned above, after diagonalising the scalar mass matrix in eq. (2.4), we get the physical masses for the two neutral scalars as

$$
\begin{aligned}
& M_{h_{1}}^{2}=\lambda_{h} v^{2}+\lambda_{D} v_{D}^{2}-\sqrt{\left(\lambda_{D} v_{D}^{2}-\lambda_{h} v^{2}\right)^{2}+\left(\lambda_{h D} v v_{D}\right)^{2}}, \\
& M_{h_{2}}^{2}=\lambda_{h} v^{2}+\lambda_{D} v_{D}^{2}+\sqrt{\left(\lambda_{D} v_{D}^{2}-\lambda_{h} v^{2}\right)^{2}+\left(\lambda_{h D} v v_{D}\right)^{2}} .
\end{aligned}
$$

We identify the lighter Higgs scalar as the SM-like Higgs observed at the LHC. Therefore, we take $M_{h_{1}}=126 \mathrm{GeV}$ and consider small mixing angle $\sin \zeta \leq 0.1$ in order to ensure agreement with the Higgs signal strengths at the LHC [68-70].

Now, we can express all the quartic couplings in terms of the physical Higgs masses as,

$$
\begin{aligned}
\lambda_{D} & =\frac{M_{h_{2}}^{2}+M_{h_{1}}^{2}+\left(M_{h_{2}}^{2}-M_{h_{1}}^{2}\right) \cos 2 \zeta}{4 v_{D}^{2}}, \\
\lambda_{h} & =\frac{M_{h_{2}}^{2}+M_{h_{1}}^{2}-\left(M_{h_{2}}^{2}-M_{h_{1}}^{2}\right) \cos 2 \zeta}{4 v_{D}^{2}}, \\
\lambda_{h D} & =\frac{\left(M_{h_{2}}^{2}-M_{h_{1}}^{2}\right) \sin 2 \zeta}{2 v v_{D}} \\
\mu_{h}^{2} & =\lambda_{h} v^{2}+\lambda_{h D} \frac{v_{D}^{2}}{2} \\
\mu_{D}^{2} & =\lambda_{D} v_{D}^{2}+\lambda_{h D} \frac{v^{2}}{2} .
\end{aligned}
$$

In the above expressions, all the quartic couplings have to be within the perturbative regime which is $\lambda_{i}<4 \pi$. 
After the $\mathrm{SU}(2)_{D}$ symmetry breaking, the DM candidate $\psi_{1}\left(=\psi_{1_{L}} \oplus \psi_{1_{R}}\right)$ will get mass which is

$$
M_{\psi_{1}}=M_{\mathrm{DM}}=\frac{\lambda_{1} v_{D}}{\sqrt{2}} .
$$

The other scalar doublet $\eta_{D}$, which has a nonzero $\mathbb{Z}_{3}$ charge, will also get mass after breaking of both SM and hidden sector gauge symmetries. In the present model, among the $\mathbb{Z}_{3}$ charged particles i.e. hidden sector fermions and scalar $\eta_{D}$, we consider the fermion $\psi_{1}$ as the lightest one. This is always possible by tuning the couplings related to $\eta_{D}$ and $\lambda_{\alpha} \mathrm{s}(\alpha=2$ to 4$)$ so that heavier $\mathbb{Z}_{3}$ charged particles decay to the lightest one and thereby $\psi_{1}$ becomes stable. Thus, $\psi_{1}$ will be a viable DM candidate in our model. Moreover, the invariance of the $\mathbb{Z}_{3}$ and $\mathbb{Z}_{2}$ symmetry actually make the hidden sector fermion mass matrix diagonal, which means unlike the quark or neutrino mixing in the SM, there is no mixing between the fermions in different $\mathrm{SU}(2)_{D}$ doublets.

\section{$3 \quad$ Neutrino mass}

In this work, as mentioned in the Model section (section 2), instead of three right handed neutrinos we consider a minimal setting and we add only two, which is sufficient to explain the current neutrino oscillation data. Therefore, in this framework, light neutrino masses are generated by the well known Type-I seesaw mechanism, where the light neutrino mass matrix is related to the Dirac and Majorana mass matrices in the following way

$$
m_{\nu}=-M_{D} M_{R}^{-1} M_{D}^{T},
$$

where $M_{D}$ is the Dirac mass matrix $(3 \times 2)$ while the Majorana mass matrix for the heavy right handed neutrinos $N_{j_{R}}(j=1,2)$ is denoted by a $2 \times 2$ matrix $M_{R}$. In this work, for simplicity and without loss of generality, we assume $M_{R}$ to be a diagonal matrix i.e. $M_{R}=\operatorname{diag}\left(M_{N_{1}}, M_{N_{2}}\right)$. One can also choose $M_{N_{1}}, M_{N_{2}}$ real and positive by redefining the phases of the spinors $N_{1}$ and $N_{2}$ in the mass eigenstate basis. Similarly, by redefining the phases of the left handed neutrinos in the flavour basis, we can remove the phases of one entire column of $M_{D}$ matrix. So we consider all the elements of the first column of the Dirac mass matrix $\left(M_{D}\right)$ as real. Therefore, in matrix form it looks like as follows,

$$
M_{D}=\frac{y_{i j} v}{\sqrt{2}}=\frac{v}{\sqrt{2}}\left(\begin{array}{ll}
y_{e e} & y_{e \mu}^{R}-i y_{e \mu}^{I} \\
y_{\mu e} & y_{\mu \mu}^{R}-i y_{\mu \mu}^{I} \\
y_{\tau e} & y_{\tau \mu}^{R}-i y_{\tau \mu}^{I}
\end{array}\right) .
$$

We have computed the physical masses of light neutrinos (eigenvalues) and mixing angles (eigenvectors) by diagonalising the complex symmetric Majorana mass matrix $m_{\nu}$. All the elements of $m_{\nu}$ are explicitly given in the appendix A. In studying the neutrino phenomenology, we take into account the observed values of neutrino oscillation parameters [71]. In this work, we mostly focus on the normal hierarchy $(\mathrm{NH})$ of the light neutrino masses, but a similar study can be done for the inverted hierarchical scenario as well. Bounds which we have considered to constrain the elements of $m_{\nu}$ matrix are two mass square differences, 
the three oscillation angles and the cosmological upper bound on the sum of three light neutrino masses. These bounds are as follows

- from the neutrino oscillation experiments there are tight constraints on two mass square differences. For NH $3 \sigma$ bounds are as follows [71],

$$
6.93 \leq \frac{\Delta m_{21}^{2}}{10^{-5}}\left(\mathrm{eV}^{2}\right) \leq 7.97 \text { and } 2.37 \leq \frac{\Delta m_{31}^{2}}{10^{-3}}\left(\mathrm{eV}^{2}\right) \leq 2.63
$$

- Three mixing angles namely, solar mixing angle $\theta_{12}$, atmospheric mixing angle $\theta_{23}$ and reactor mixing angle $\theta_{13}$ are also very well measured now. The allowed values of mixing angles for $\mathrm{NH}$ in $3 \sigma$ are listed below [71]

$$
\begin{aligned}
30^{0} & \leq \theta_{12} \leq 36.51^{0} \\
7.82^{0} & \leq \theta_{13} \leq 9.02^{0} \\
37.99^{0} & \leq \theta_{23} \leq 51.71^{0}
\end{aligned}
$$

- From the Planck data [17] there is a bound on the sum of light neutrinos masses which is $\sum_{i=1,2,3} m_{\nu_{i}} \leq 0.23 \mathrm{eV}$.

Moreover, we have not applied any bound on the $\mathrm{CP}$ violating phase $\delta$, which is yet to be measured accurately by the different ongoing and upcoming oscillation experiments like T2K [72], T2HK [73], DUNE [74-77] and INO [78]. There is a hint of maximal CP violation $\left(\delta \sim-\frac{\pi}{2}\right)$ from the T2K experiment [79], which excludes the CP conserving values of $\delta=0$ or $\pi$, at $90 \%$ C.L. However, there is some tension between the observed value of $\delta$ from T2K and NOvA [80].

In satisfying the above mentioned neutrino oscillation parameters, we vary the model parameters, specifically, the elements of the Dirac mass matrix (eq. (3.2)) and the RHneutrino masses in the following range,

$$
\begin{aligned}
10^{-4} & \leq y_{e e}, y_{\mu e}, y_{\tau e}, y_{e \mu}^{R} \leq 10^{-2}, \\
10^{-3} & \leq y_{\mu \mu}^{R}, y_{\mu \mu}^{I}, y_{\tau \mu}^{R}, y_{\tau \mu}^{I} \leq 1, \\
10^{-6} & \leq y_{e \mu}^{I} \leq 10^{-4}, \\
10^{8}\left(10^{13}\right) & \leq M_{N_{1}}\left(M_{N_{2}}\right) \mathrm{GeV} \leq 10^{11}\left(10^{15}\right) .
\end{aligned}
$$

In eq. (3.2), all the elements in the first column are real and positive (can be made by phase rotation) while the elements in the second column can have both positive as well as negative values. However, to make the $\mathrm{CP}$ asymmetry parameter positive we consider only positive values of all the elements in the second column of the Dirac mass matrix, i.e. in the above $y_{\alpha \mu}^{R, I}=\left|y_{\alpha \mu}^{R, I}\right|,(\alpha=e, \mu, \tau)$. Here, we take hierarchical RH-neutrino masses, which is evident from the ranges of $M_{N_{1}}$ and $M_{N_{2}}$ that we have chosen in eq. (3.5). The hierarchical scenario for the RH-neutrino masses implies that we have to consider the lepton as well as DM asymmetry generated from the decay of lightest RH-neutrino $\left(N_{1}\right)$ only. Indeed the asymmetry generated from the decay of $N_{2}$ will be washed out by the decay as well as 
inverse decay of $N_{1}$ which is still in thermal equilibrium during $T \sim M_{N_{2}}$. In the result section (section 5), we will see that there exist correlations among the parameters of the neutrino sector and hence not the entire adopted ranges in eq. (3.5) are allowed by the neutrino oscillation data and the requirement of successful leptogenesis.

\section{Boltzmann equation for studying lepton asymmetry and Dark Matter asymmetry}

As we have already mentioned earlier, in this work our goal is to generate an asymmetry in the dark sector following the idea of leptogenesis in the visible sector. In other words, the asymmetries in both sectors may have a common source i.e. they can be generated from the CP-violating out-of-equilibrium decay of the lightest RH-neutrino $N_{1}$. Therefore, we need to solve (at least) three Boltzmann equations simultaneously: one gives the number density for the lightest RH-neutrino $N_{1}$ and the other two will govern the asymmetries of the visible and dark sectors. As mentioned in [29], depending on the decay width and the mass of the RH neutrinos, the source of dark and visible sector asymmetry production can be divided in to two regimes. In the case $\Gamma_{N_{1}} \ll M_{N_{1}}$, we are in the narrow width approximation and the $\mathrm{RH}$ neutrino couples very weakly to the thermal bath, so that it is strongly out of equilibrium. On the other hand, for $\Gamma_{N_{1}} \simeq M_{N_{1}}$, we are in the large washout or transfer regime where the wash-out processes mediated by $N_{1}$ are not negligible. In the current work, we mostly focus on the RH-neutrino mass around $10^{9} \mathrm{GeV}$ and from the light neutrino mass constraint which is $\sim 10^{-11} \mathrm{GeV}$ we have very small Yukawa couplings in the Dirac mass, hence we are in the narrow width approximation regime with $\Gamma_{N_{1}} \ll M_{N_{1}}$. Moreover, for this assumption we neglect the transfer diagrams [29].

The relevant Boltzmann equations which we need to solve for the narrow width approximation regime are as follows,

$$
\begin{aligned}
\frac{d Y_{N_{1}}}{d z} & =-\frac{z}{s H\left(M_{1}\right)}\left[\left(\frac{Y_{N_{1}}}{Y_{N_{1}}^{e q}}-1\right) \times\left(\gamma_{D_{1}}+2 \gamma_{\phi, s}^{1}+4 \gamma_{\phi, t}^{1}\right)\right] \\
\frac{d Y_{\Delta l}}{d z} & =-\frac{\Gamma_{N_{1}}}{H\left(M_{1}\right)}\left[\epsilon_{l} \frac{z K_{1}(z)}{K_{2}(z)}\left(Y_{N_{1}}^{e q}-Y_{N_{1}}\right)+\operatorname{Br}_{l} \frac{z^{3} K_{1}(z)}{4} Y_{\Delta l}\right], \\
\frac{d Y_{D}}{d z} & =-\frac{\Gamma_{N_{1}}}{H\left(M_{1}\right)}\left[\epsilon_{D} \frac{z K_{1}(z)}{K_{2}(z)}\left(Y_{N_{1}}^{e q}-Y_{N_{1}}\right)+\operatorname{Br}_{D} \frac{z^{3} K_{1}(z)}{4} Y_{D}\right],
\end{aligned}
$$

where the first equation represents the evolution of the comoving yield $Y_{N_{1}}$ of $N_{1}$. The yield of a species is defined as the actual number density of that species divided by the entropy density of the Universe. If there is no interaction then the yield of a species remains unaltered, as the expansion of the Universe dilutes the number density and the entropy density in the same way.

The R.H.S. of the Boltzmann equation for $N_{1}$ describes the possible ways to change the number density of $N_{1}$. The quantity $\gamma_{D 1}$ is related to the total decay width of $N_{1}$ i.e. the decay of $N_{1}$ into both visible and dark sectors. On the other hand, $\gamma_{\phi, s}^{1}$ and $\gamma_{\phi, t}^{1}$ are related to $s$-channel and $t$-channel scattering of $N_{1}$ mediated by $\phi_{h}$, which can also lead to the destruction or production of $N_{1}$. The expressions of $\gamma_{D_{1}}, \gamma_{\phi_{h}, s}^{1}$ and $\gamma_{\phi_{h}, t}^{1}$ are given in 
the appendix B. The second and third equations are the evolution equations for the lepton asymmetry and Dark Matter asymmetry, respectively. The first term in the R.H.S. of eq. (4.2) (eq. (4.3))) is the source term of lepton (Dark Matter) asymmetry from $N_{1}$ decay, while the second term represents the washout effects on the created lepton (Dark Matter) asymmetry due to the inverse decays of $N_{1}$. In eqs. (4.2), (4.3), $\mathrm{Br}_{l}$ and $\mathrm{Br}_{D}$ are the branching ratios of $\mathrm{RH}$ neutrino $N_{1}$ decay to leptonic sector and dark sector, respectively. In the above equations the $\mathrm{CP}$ asymmetry parameters $\epsilon_{l, D}$ are zero at tree level. However, by considering both tree level and one loop level diagrams (vertex correction and wave function correction, see figure 2 of [82]), non-zero values for the CP-asymmetry parameters $\epsilon_{l, D}$ arise due to the interference between tree level and one loop level diagrams. The CP asymmetry parameter for the visible sector is defined as [25, 29]

$$
\begin{aligned}
\epsilon_{l} & =\frac{\Gamma\left(N_{1} \rightarrow L \phi_{h}\right)-\Gamma\left(N_{1} \rightarrow \bar{L} \phi_{h}^{\dagger}\right)}{\Gamma_{N_{1}}}, \\
& =\frac{M_{N_{1}}}{16 \pi M_{N_{2}}} \frac{\operatorname{Im}\left[3\left(\left(y^{\dagger} y\right)_{12}^{\star}\right)^{2}+2 \alpha_{1}^{\star} \alpha_{2}\left(y^{\dagger} y\right)_{12}^{\star}\right]}{\left[\left(y^{\dagger} y\right)_{11}+\alpha_{1} \alpha_{1}^{\star}\right]},
\end{aligned}
$$

where we have normalized to the total RH neutrino decay rate and summed over the lepton flavours, i.e. $\epsilon_{l}=\sum_{\alpha} \epsilon_{l}^{\alpha}$. In eq. (4.4) we include contributions from the vertex and wavefunction diagrams with virtual SM states as in classic leptogenesis, see e.g. [25], and also the contribution from the wave-function diagram with virtual dark sector states.

Similarly, the CP asymmetry in dark sector is defined as [29]

$$
\begin{aligned}
\epsilon_{D} & =\frac{\Gamma\left(N_{1} \rightarrow \psi_{1 L} \eta_{D}\right)-\Gamma\left(N_{1} \rightarrow \overline{\psi_{1 L}} \eta_{D}^{\dagger}\right)}{\Gamma_{N_{1}}}, \\
& =\frac{M_{N_{1}}}{16 \pi M_{N_{2}}} \frac{\operatorname{Im}\left[2 \alpha_{1}^{\star} \alpha_{2}\left(y^{\dagger} y\right)_{12}^{\star}+3\left(\alpha_{1}^{\star} \alpha_{2}\right)^{2}\right]}{\left[\left(y^{\dagger} y\right)_{11}+\alpha_{1} \alpha_{1}^{\star}\right]} .
\end{aligned}
$$

In the case of Dark Matter, we have in an analogous way included contributions from the vertex and wave-function diagrams from the dark sector and only the wave-function diagram from the leptons. The total decay width of the RH-neutrino $N_{1}$ is given by

$$
\Gamma_{N_{1}}=\frac{M_{N_{1}}}{8 \pi}\left[\left(y^{\dagger} y\right)_{11}+\left|\alpha_{1}\right|^{2}\right] .
$$

From eqs. (4.4), (4.5) we see that both $\epsilon_{l}$ and $\epsilon_{D}$ are determined by the Yukawa couplings $y_{i j}$ and $\mathrm{RH}$-neutrino masses. One very important thing to stress again is that in the dark sector we can absorb the phases of the couplings $\alpha_{j}(j=1,2)$ by redefining the phases of $\psi_{1 L}$ and the complex scalar doublet $\eta_{D}$. Indeed, as in the case of a single generation of fermions, no CP phase is physical in the DM sector. Therefore the only source of CP violation for both the leptonic and DM sectors are the imaginary parts of the lepton-neutrino Yukawa couplings. We have then

$$
\frac{\epsilon_{l}}{\epsilon_{D}}=\frac{\operatorname{Im}\left[3\left(\left(y^{\dagger} y\right)_{12}^{\star}\right)^{2}+2 \alpha_{1} \alpha_{2}\left(y^{\dagger} y\right)_{12}^{\star}\right]}{2 \alpha_{1} \alpha_{2} \operatorname{Im}\left[\left(y^{\dagger} y\right)_{12}^{\star}\right]}=1+\frac{\operatorname{Im}\left[3\left(\left(y^{\dagger} y\right)_{12}^{\star}\right)^{2}\right]}{2 \alpha_{1} \alpha_{2} \operatorname{Im}\left[\left(y^{\dagger} y\right)_{12}^{\star}\right]}=1+3 \frac{\sum_{\beta} y_{\beta e} y_{\beta \mu}^{R}}{\alpha_{1} \alpha_{2}} .
$$


We see that in the particular case when $\left(y^{\dagger} y\right)_{12}^{\star}$ is purely imaginary, i.e. $y^{R}=0$, or generically when $\alpha_{1} \alpha_{2} \gg \sum_{\beta} y_{\beta e} y_{\beta \mu}^{R}$, the two $\mathrm{CP}$ violation parameters are equal and we can expect a similar asymmetry in the two sectors, as long as the wash-out processes are negligible. This is indeed not difficult to achieve as the Yukawas connected to the first generation of the SM $y_{\beta e}$ have to be small to give the small mixing angle $\theta_{13}$. From the matrix in eq. (3.2), we have that

$$
\left(y^{\dagger} y\right)_{12}^{\star}=y_{e e}\left(y_{e \mu}^{R}+i y_{e \mu}^{I}\right)+y_{\mu e}\left(y_{\mu \mu}^{R}+i y_{\mu \mu}^{I}\right)+y_{\tau e}\left(y_{\tau \mu}^{R}+i y_{\tau \mu}^{I}\right)
$$

so that this quantity is purely imaginary when the second column of the Dirac mass matrix is purely imaginary and only six real Yukawa parameters remain. Note that in this limit, the $\mathrm{CP}$ violation in the $\mathrm{RH}$ neutrino decay can still be large, while the light neutrino mass matrix is real and the Dirac phase is therefore vanishing. We have checked that with only imaginary components in the second column of the Dirac mass matrix (see eq. (3.2)) and also in the same range of the parameters value as given in eq. (3.5), one can easily obtain the three neutrino mixing angles and the two mass square differences in their observed $3 \sigma$ ranges. For this particular choice of parameters, one can easily estimate the value of the lepton CP asymmetry parameter $\left(\epsilon_{l}\right)$. In this case $\epsilon_{l}$ takes the following form,

$$
\epsilon_{l}=\frac{M_{N_{1}}}{8 \pi M_{N_{2}}} \frac{\alpha_{2}}{\alpha_{1}} \operatorname{Im}\left[\left(y^{\dagger} y\right)_{12}^{\star}\right]
$$

for large $\alpha_{2} / \alpha_{1}$ and $\left(y^{\dagger} y\right)_{11}<\alpha_{1}^{2}$. If we take $\operatorname{Im}\left(y^{\dagger} y\right)_{12}^{\star} \sim 2 \times 10^{-3}, M_{N_{1}} / M_{N_{2}} \sim 10^{-4}$ and $\alpha_{2} / \alpha_{1} \sim 10^{2}$, then we obtain $\epsilon_{l} \sim 10^{-6}$ sufficient to generate the lepton asymmetry of the Universe, as we will show later. One important conclusion we can draw for this scenario is that although there is no low scale CP violation, ${ }^{3}$ there still exist a sufficiently large high scale $\mathrm{CP}$ violation by which we can generate lepton asymmetry of the Universe and henceforth the observed baryon asymmetry of the Universe.

Another limiting case is when the real and imaginary parts $y_{i \mu}^{R, I}$ in the second column of the Yukawa matrix are equal and large. In that case it is $\left(\left(y^{\dagger} y\right)_{12}^{\star}\right)^{2}$ which becomes purely imaginary and can even dominate the $\mathrm{CP}$ violation in the leptonic sector. In that case the dark CP violation parameter $\epsilon_{D}$ can be substantially smaller, but we can compensate the smaller number density of the Dark Matter by increasing its mass and still satisfy the CMB constraint. Moreover, note that we can take large value of $\alpha_{2}(\sim \mathcal{O}(1))$ without violating any constraint, but a large value of $\alpha_{1}$ will violate the narrow width approximation $\left(\Gamma_{N_{1}} \ll M_{N_{1}}\right.$ ) via eq. (4.6). Therefore, remaining within the narrow width approximation, but at the same time aiming to increase the CP asymmetry parameter $\epsilon_{l}$ for the production of sufficient lepton asymmetry, we vary the two parameters $\alpha_{1}$ and $\alpha_{2}$ in the following ranges:

$$
\begin{aligned}
10^{-3} & \leq \alpha_{1} \leq 10^{-2} \\
0.3 & \leq \alpha_{2} \leq 1.0 .
\end{aligned}
$$

\footnotetext{
${ }^{3}$ If the light neutrino mass matrix is real, the Dirac phase $\delta$ is vanishing, but the Majorana phases can still be non-trivial if the eigenvalues of the mass matrix have different sign. This indeed happens if one column of the Yukawa matrix is imaginary, see the discussion in appendix A.
} 
By solving the above three Boltzmann equations, we obtain the lepton and DM asymmetries as a function of the temperature of the Universe. For leptogenesis, we need to calculate the lepton asymmetry before the sphaleron decoupling temperature $T_{s p h} \sim 150 \mathrm{GeV}[81,82]$ because the produced lepton asymmetry has to be converted into the observed baryon asymmetry via sphaleron transitions in equilibrium. The conversion factor from $Y_{\Delta l}$ to $Y_{B}$ is given by [83]

$$
Y_{B}=\frac{8 N_{f}+4 N_{\phi_{h}}}{22 N_{f}+13 N_{\phi_{h}}} Y_{\Delta l}
$$

where $N_{f}$ is number of generations of quarks and leptons while $N_{\phi_{h}}$ being number of scalar doublets in the visible sector.

Regarding the Dark Matter, we need to ensure that the symmetric DM component efficiently annihilates away leaving only the asymmetric component. As the Dark Matter is charged under a Dark $\mathrm{SU}(2)$ gauge group, this is not difficult to achieve as long as the Dark Matter mass is not too large. Indeed the dominant annihilation channel for an $\mathrm{SU}(2)$ doublet is into the corresponding gauge bosons, that subsequently annihilate/decay through the scalar sector into SM states. ${ }^{4}$ The generic expectation for the annihilation rate of a doublet is similar to that of the Higgsino in supersymmetric models and it is enhanced by coannihilations [84] and even the Sommerfeld effect. In the case of Dark Matter mass of similar order to the gauge boson mass and $\lambda_{2} \gtrsim \lambda_{1}$, the coannihilation effect is dominant and we obtain [85]

$$
\sigma_{\psi \bar{\psi} \rightarrow W_{D} W_{D}} \sim \frac{g_{D}^{4}}{128 \pi M_{\mathrm{DM}}^{2}}=2.5 \times 10^{-9} \mathrm{GeV}^{-2} g_{D}^{4}\left(\frac{M_{\mathrm{DM}}}{1 \mathrm{TeV}}\right)^{-2}
$$

which is slightly larger than the thermal cross section $\langle\sigma v\rangle \sim 10^{-9} \mathrm{GeV}^{-2}$. So for a Dark Gauge coupling of order one, the symmetric Dark Matter component becomes important only at masses above $1 \mathrm{TeV}$. Even heavier masses can be allowed if the gauge bosons remain light and the Sommerfeld effect increases the cross section further [86].

For masses below the $\mathrm{TeV}$ range, where the annihilation is strong enough, we can determine the Dark Matter relic density by computing Dark Matter asymmetry at the present epoch $T=T_{0} \sim \mathcal{O}\left(10^{-13}\right) \mathrm{GeV}(z \rightarrow \infty)$ and using the following relation [84],

$$
\Omega h^{2}=2.755 \times 10^{8}\left(\frac{M_{\mathrm{DM}}}{\mathrm{GeV}}\right) Y_{D}(z \rightarrow \infty) .
$$

Earlier WMAP and now the Planck satellite have measured the DM relic density very precisely and its present value at the $68 \%$ C.L. is [17]

$$
0.1172 \leq \Omega h^{2} \leq 0.1226
$$

Equating eq. (4.13) and eq. (4.14) one can find the mass range for the Dark Matter particle $M_{\mathrm{DM}}$ which reproduces the observed Dark Matter abundance.

\footnotetext{
${ }^{4}$ In the same way, the symmetric component of $\psi_{3}$ annihilates away.
} 


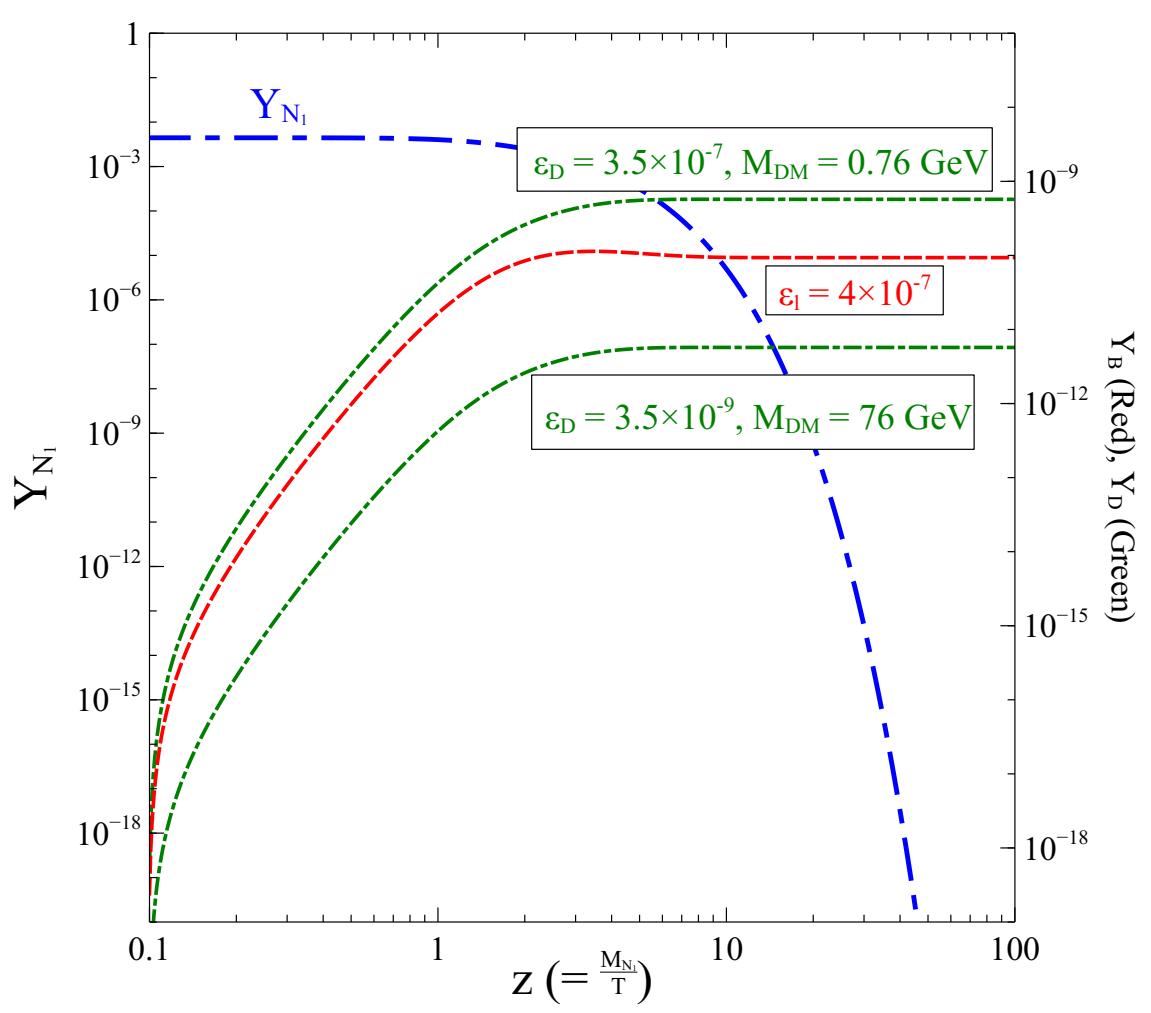

Figure 1. Variation of baryon asymmetry of the Universe and the dark sector asymmetry with z. In generating the figure we have assumed the following values $M_{N_{1}}=10^{10} \mathrm{GeV}, M_{N_{2}}=10^{13} \mathrm{GeV}$, $y^{\dagger} y=10^{-6}, \Gamma_{N_{1}} \sim 398 \mathrm{GeV}, B r_{\chi}=0.2, B r_{l}=0.8$.

Let us finally comment on the presence of the inert doublet $\eta_{D}$, which is also produced in the $\mathrm{RH}$ neutrino decay and, as it is heavier, could regenerate a $\psi_{1}$ population after freeze-out and after the $\mathrm{SU}(2)_{D}, \mathrm{SU}(2)_{E W}$ gauge symmetries are broken, by decaying into a DM fermion and a lepton. The number density of $\eta_{D}$ can be efficiently reduced by the cubic interaction with $\phi_{D}$, which allows for semiannihilation [87] through the process $\eta_{D}+\eta_{D} \rightarrow \eta_{D}^{\star}+h_{1,2}$. In this case semiannihilation is determined by the potential parameter $\lambda_{D 3}$, which does not affect the stability of the vacuum and can be chosen large to allow for a sufficiently large semiannihilation cross section. We assume here that due to such process only a negligible number density of the inert doublet is left after freeze-out.

\section{Results}

In figure 1, we show the production of the baryon asymmetry and the Dark Matter asymmetry from the decay of $\mathrm{RH}$ neutrino $N_{1}$. When the $\mathrm{RH}$ neutrino starts decaying, both the lepton sector asymmetry and the dark sector asymmetry grow fast to their final values. For $\epsilon_{l}=4 \times 10^{-7}$ and taking the other parameters values as mentioned in the caption, we obtain the correct value of the matter antimatter asymmetry of the Universe which lies within the value measured by the Planck satellite. For two values of $\epsilon_{D}=3.5 \times 10^{-7}$ and $3.5 \times 10^{-9}$, we can provide for a dark sector asymmetry which fulfils the total Dark Matter 

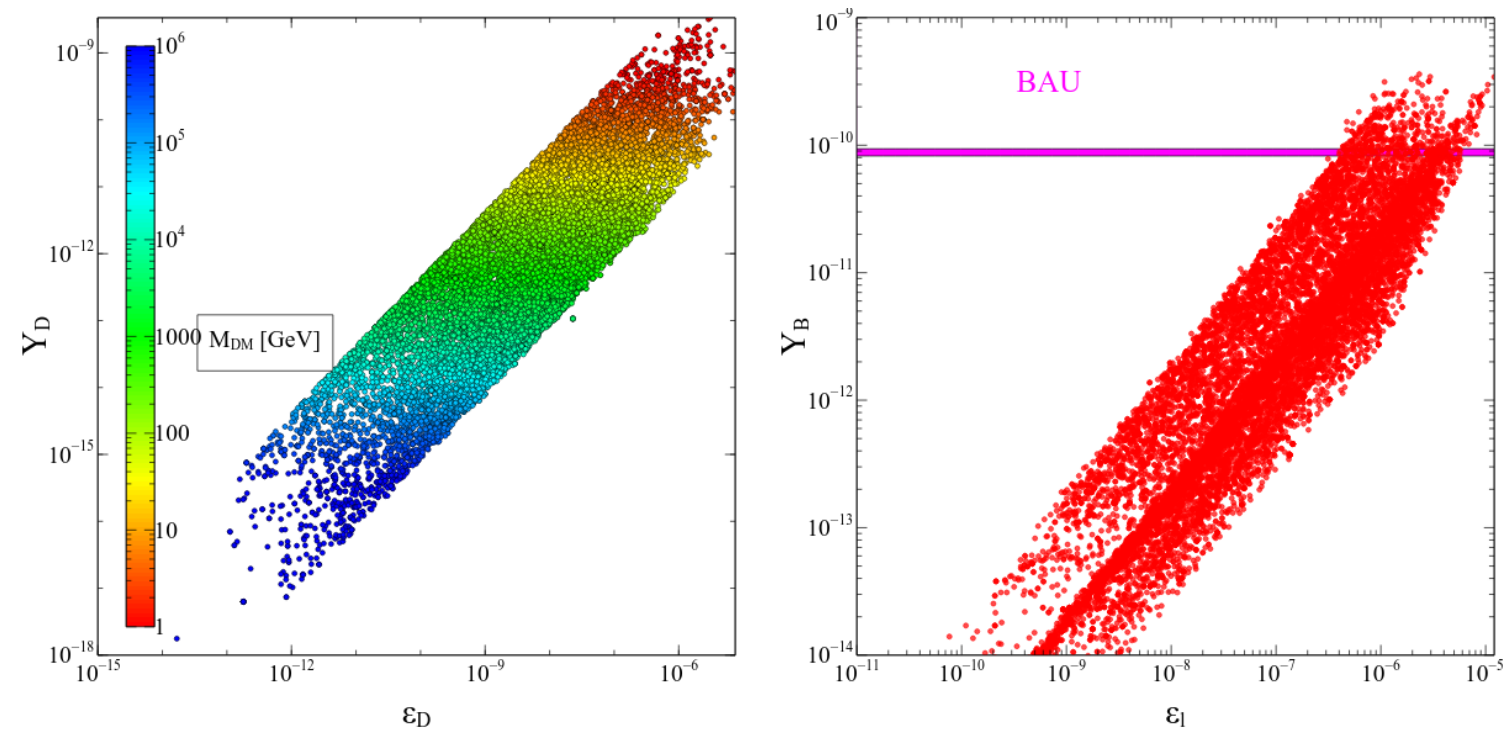

Figure 2. Left panel (LP) shows the variation in the $Y_{D}-\epsilon_{D}$ plane and in the right panel (RP) variation in $\left(Y_{B}-\epsilon_{l}\right)$ plane has been shown. All the points satisfy neutrino oscillation data as mentioned in section 3. All the parameters have been varied in the range as shown in section 3 .

abundance for a Dark Matter mass of $0.76 \mathrm{GeV}$ and $76 \mathrm{GeV}$, respectively. One interesting thing to note here is that for $\epsilon_{D}=3.5 \times 10^{-7}$ (which is less than $\epsilon_{l}$ ) we are producing more dark asymmetry than the lepton asymmetry. This is because we have considered $B r_{D}<B r_{l}$ i.e washout effects for the dark sector are weaker than for the visible sector. So even for CP violation of the same order, we can obtain an enhancement of the Dark Matter density compared to the baryon density giving naturally $\Omega_{\mathrm{DM}}>\Omega_{b}$ for DM masses in the range of the charm/bottom quark mass. In the subsequent figures we will see the dependence of the baryon and dark sector asymmetry on the model parameters and how they are related with neutrino oscillations.

In the LP of figure 2, we show the variation of the Dark Matter yield with $\epsilon_{D}$. All the points satisfy the neutrino oscillation data. The figure shows a sharp correlation between $\epsilon_{D}$ and $Y_{D}$, as expected in the narrow width regime. For the particular value of the yield we determine the Dark Matter mass such that the DM energy density coincides with the observed value using the expression as given in eq. (4.13). We see that the present model can accommodate the right Dark Matter abundance in the mass range $M_{\mathrm{DM}}=1-10^{6} \mathrm{GeV}$, which is, at least in the lower mass range, within the sensitivity of ongoing direct detection experiments. The large mass region above the $\mathrm{TeV}$ is disfavoured by the fact that the $\mathrm{CP}$ violation parameter has to be very suppressed and by the possible presence of a substantial symmetric DM component.

In the RP, we show the allowed region in the $Y_{B}-\epsilon_{l}$ plane. As expected, here also a direct correlation exist between these two quantities. The narrow magenta band is the present day accepted value of the baryon anti-baryon asymmetry of the Universe. The parameter values for which the baryon asymmetry lies above the magenta line are ruled out, while below the line leptogenesis cannot provide the full matter-antimatter asymmetry 

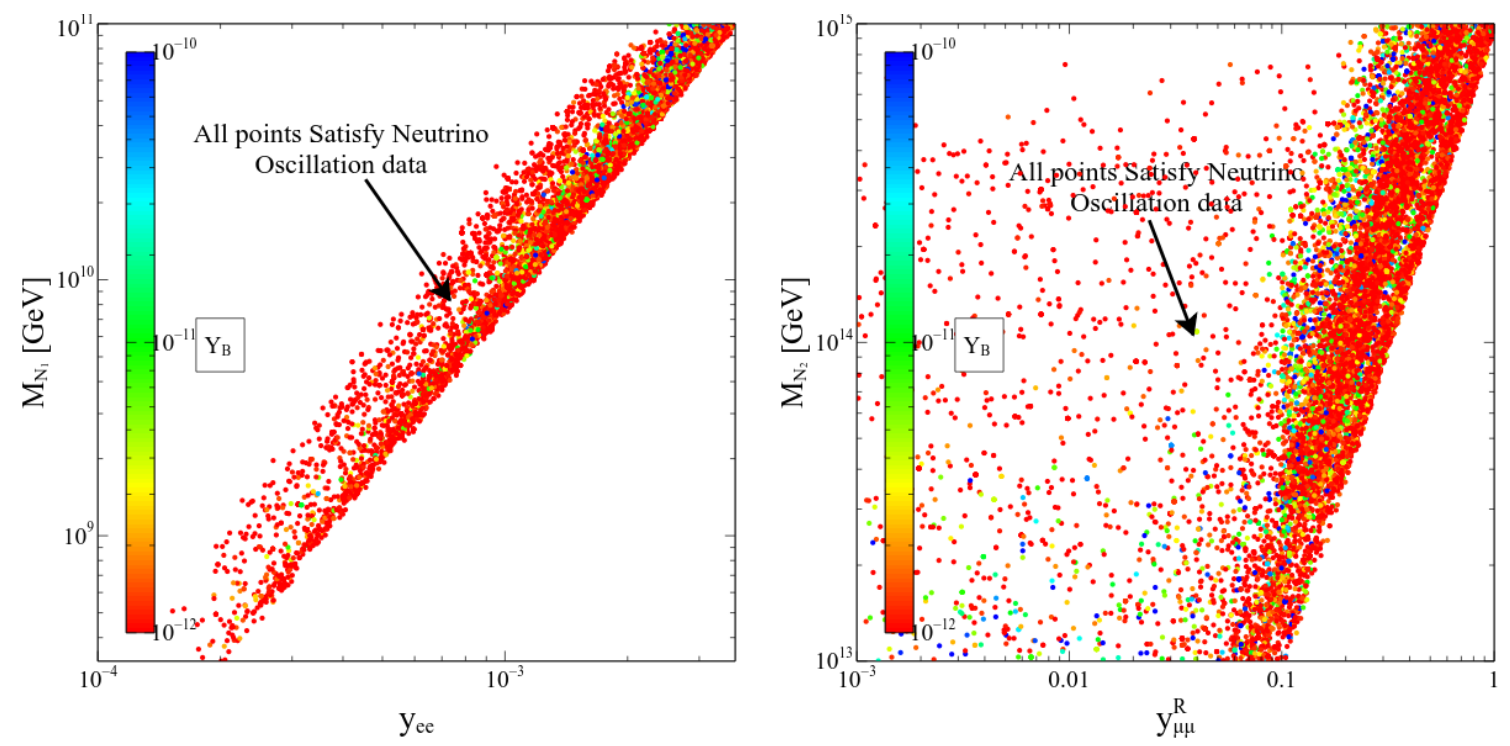

Figure 3. LP (RP): scatter plot in the $y_{e e}-M_{N_{1}}\left(y_{\mu \mu}^{R}-M_{N_{2}}\right)$ plane after satisfying neutrino oscillation data as mentioned in section 3. All the parameters have been varied in the range as shown in section 3 .

of the Universe. We see that we need a CP violation parameter $\epsilon_{l} \sim 10^{-6}$ in order to obtain the observed baryon asymmetry. Moreover, comparing the LP with the RP, it is clear that we can indeed achieve the right abundance of Dark Matter and baryons for the case $\epsilon_{l} \sim \epsilon_{D}$ and then the Dark Matter mass is in the range $1-10 \mathrm{GeV}$ as expected.

In the LP and RP of figure 3, we show the correlation among the RH neutrino masses and the elements of the Dirac mass matrix, which is required in order to satisfy the oscillation data. We see that the ratio $\frac{y_{e e}^{2}}{M_{N_{1}}}$ is fixed by neutrino oscillations, as it provides one of the mass scales in the light neutrino mass matrix. Similar correlations of $M_{N_{1}}$ are present also for the other Yukawa parameters in the first column, $y_{\mu e}, y_{\tau e}$. Here we are generating the neutrino mass by the Type-I seesaw mechanism, hence, the elements in the first column of the Dirac mass matrix (see eq. (3.2) and appendix A) are directly related to $M_{N_{1}}$. Similarly, the elements of the second column of the Dirac mass matrix are always suppressed by $1 / M_{N_{2}}$ when they appear in the light neutrino mass matrix. Therefore, for the RP we also see a similar kind of correlation with elements of the second column of Dirac mass matrix and $M_{N_{2}}$, but in this case the correlation is less sharp. Since the RH neutrinos $N_{1}$ and $N_{2}$ are hierarchical, a difference in the magnitude of the elements of the first and second column of the Yukawa matrix are arranged such that both the light neutrino mass squared differences we obtain are consistent with the neutrino oscillation data. In these plots, all points are allowed by neutrino oscillation data and some of them (indicated by blue colour) produce the observed matter-antimatter asymmetry of the Universe and for rest we need extra sources of baryogenesis. Indeed we have adjusted the imaginary part of the elements of the second column of the Yukawa matrix such that both the lepton asymmetry as well as the light neutrino mass matrix are obtained, consistent with their respective measured values. 

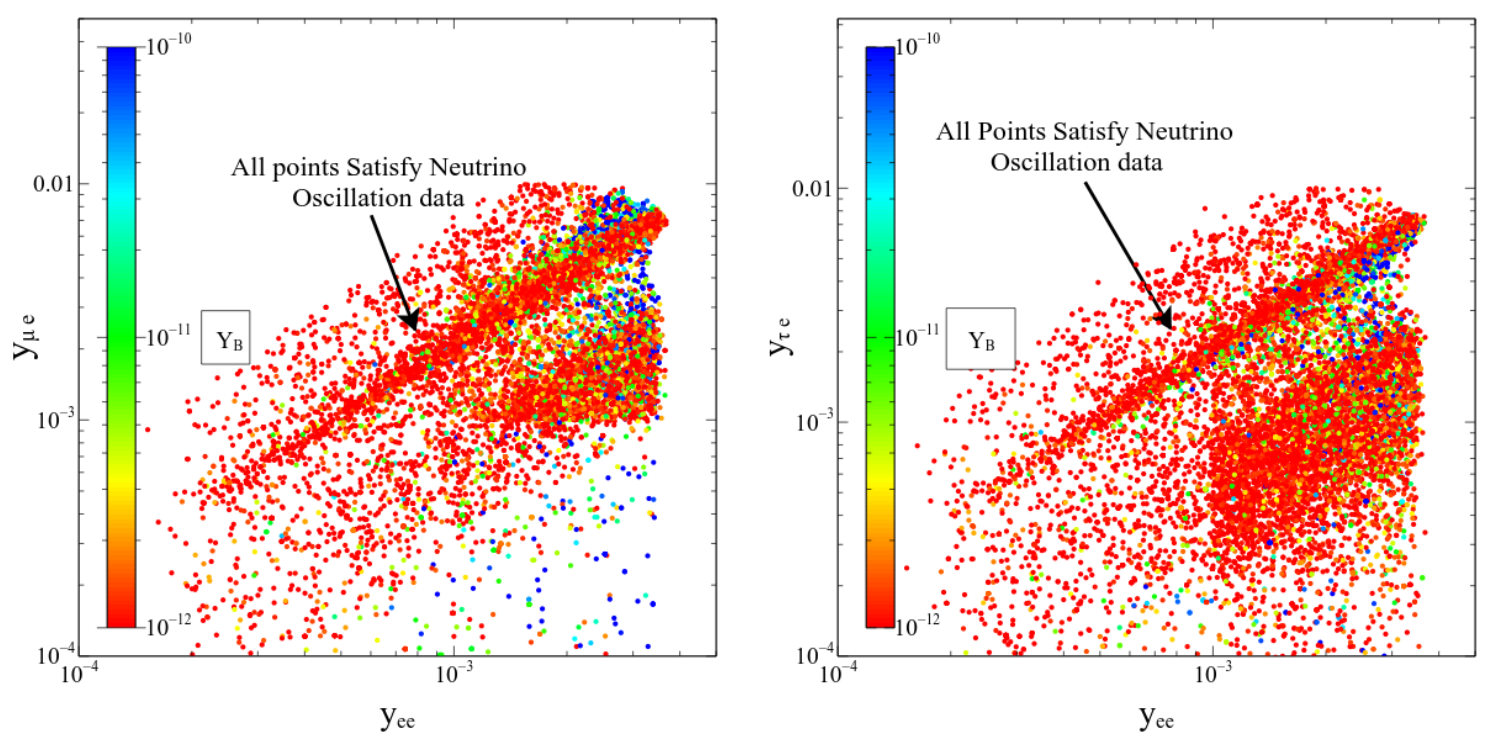

Figure 4. LP (RP): scatter plot in the $y_{e e}-y_{\mu e}\left(y_{e e}-y_{\tau e}\right)$ plane after satisfying neutrino oscillation data as mentioned in section 3. All the parameters have been varied in the range as shown in section 3 .
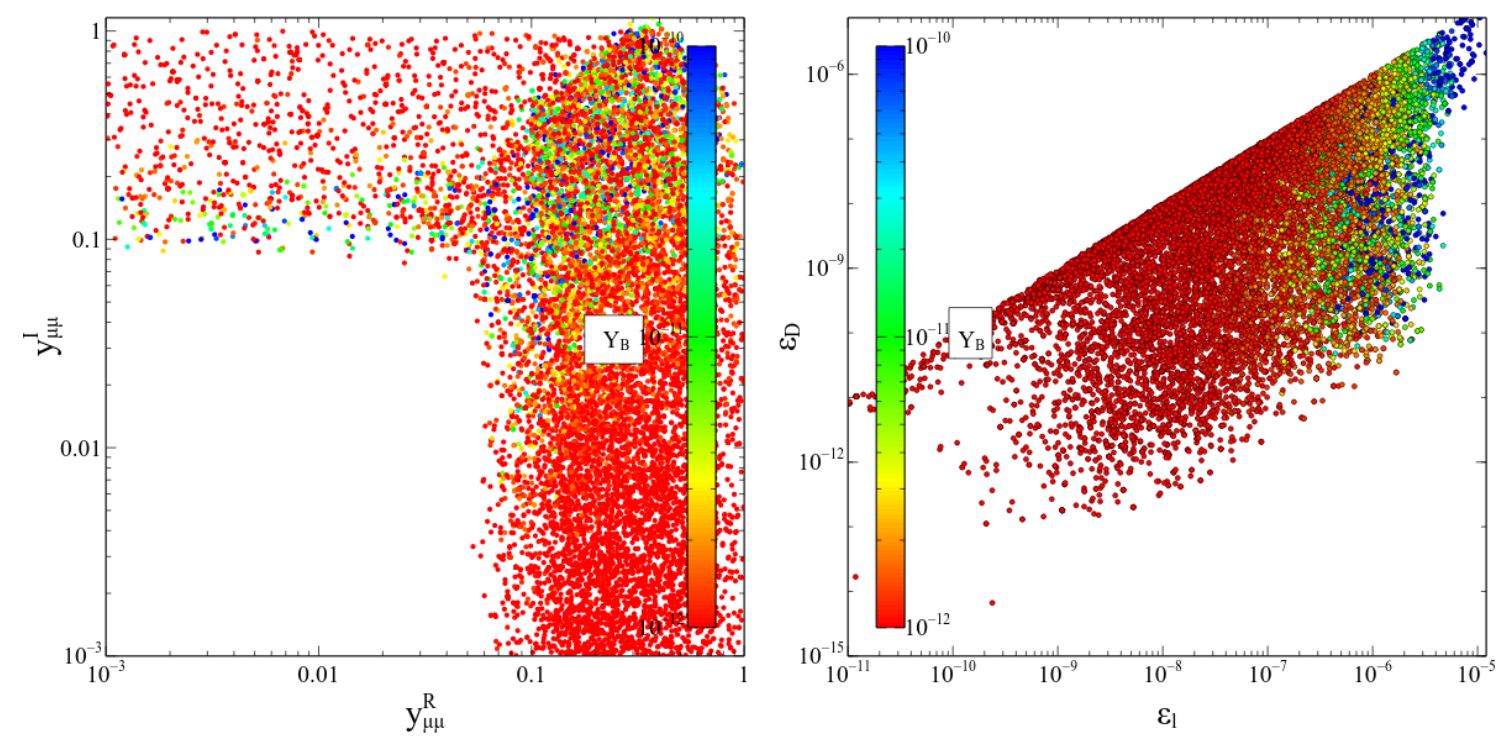

Figure 5. LP (RP): scatter plot in the $y_{\mu \mu}^{R}-y_{\mu \mu}^{I}\left(\epsilon_{l}-\epsilon_{D}\right)$ plane after satisfying neutrino oscillation data as mentioned in section 3. All the parameters have been varied in the range as shown in section 3 .

In figure 4, we show the allowed region after satisfying the neutrino oscillation data in both LP and RP. A clear correlation exist among the parameters in order to satisfy neutrino oscillation data. Only the blue points are close to the current value of the Universe's baryon asymmetry, while the red and green points give a too low value.

The LP of figure 5 shows explicitly the correlation between the real and imaginary part of the same element of the Dirac mass matrix, i.e. $y_{\mu \mu}^{R}$ and $y_{\mu \mu}^{I}$. Either of them can give 


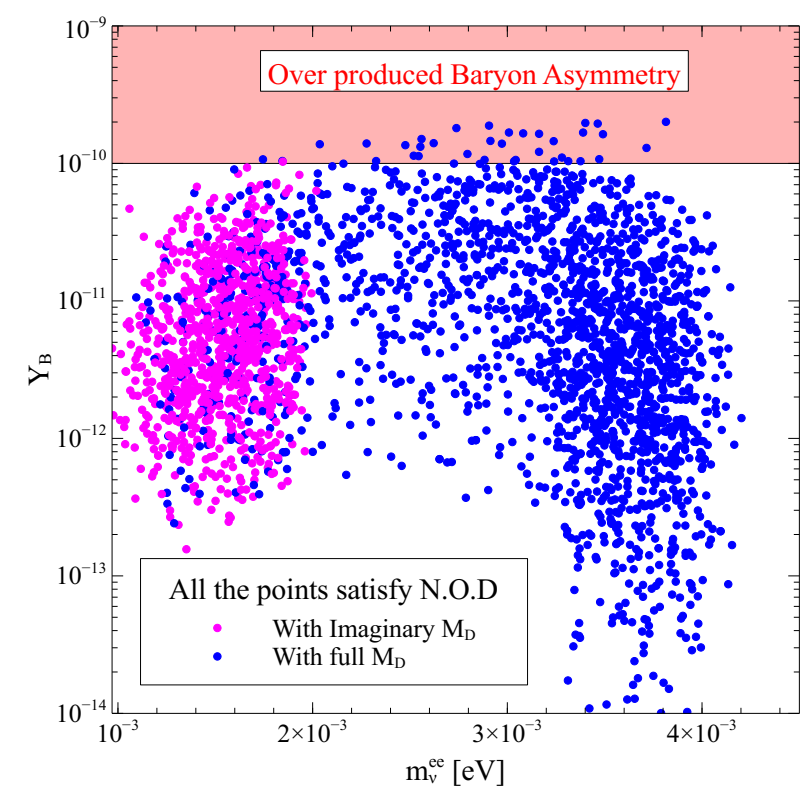

Figure 6. Scatter plot in the $m_{\nu}^{e e}-Y_{B}$ plane after satisfying neutrino oscillation data (N.O.D) as mentioned in section 3. All the parameters have been varied in the range as shown in section 3 .

the right contribution to the light neutrino mass matrix to fit the oscillation data, but only a substantial imaginary part allows for non-vanishing $\mathrm{CP}$ violation and the production of a sufficiently large lepton asymmetry. This plot shows that the correct baryon asymmetry can be obtained both if the real and imaginary part are equal and large or when the real part is negligible and the imaginary part provides a substantial contribution to both the $\mathrm{CP}$ violation and the neutrino mass. This two cases correspond to the limiting cases discussed earlier.

In $\mathrm{RP}$ of the same figure we show the allowed region in the $\epsilon_{l}-\epsilon_{D}$ plane. We see that our predicted baryon asymmetry comes close to the measured value only for reasonably high values of $\epsilon_{l}$. Since there exists a sharp correlation between $Y_{B}$ and $\epsilon_{l}$, lower $\epsilon_{l}$ results in production of lower lepton asymmetry. As we discussed earlier, we expect $\epsilon_{D}$ to be equal or less than $\epsilon_{l}$ and indeed this is also reproduced in this figure. Note that while the baryon asymmetry is correctly given only in a quite narrow region of the parameter space, a much wider range of $\epsilon_{D}$ is allowed as we can adjust the DM mass to match the Dark Matter abundance. Generically the mass of the Dark Matter state is given by the VEV of the Dark $\mathrm{SU}(2)_{D}$ scalar doublet and can vary compared to the scale of SM fermion masses. Nevertheless a natural range for the mechanism to work are DM masses in the GeV to tens of $\mathrm{GeV}$ range, well below the WIMP mass scale around $1 \mathrm{TeV}$.

In figure 6, we give the allowed model points in the $m_{\nu}^{e e}-Y_{B}$ plane. Both the blue and magenta points satisfy fully the neutrino oscillation data and the only difference between them is the choice of the Dirac mass matrix elements in eq. (3.2). The blue points correspond to the general $M_{D}$ matrix with complex second column, whereas the magenta points are obtained for a purely imaginary second column of $M_{D}$ (i.e. $y_{i \mu}^{R}=0, i=e, \mu, \tau$ ). Taking the Majorana phase angle convention for the light neutrinos as $\operatorname{diag}\left(1, e^{i \alpha_{21} / 2}, e^{i \alpha_{31} / 2}\right)$ and 
one zero mass eigenstate $m_{1}=0$, we can write down the $(1,1)$ element of the neutrino mass matrix $\left(m_{\nu}\right)$, which generates the $0 \nu \beta \beta$ decay, as

$$
m_{\nu}^{e e}=\left[s_{12}^{4} c_{13}^{4} m_{2}^{2}+2 m_{2} m_{3} s_{12}^{2} c_{13}^{2} s_{13}^{2} \cos \left(\alpha_{31}-\alpha_{21}-2 \delta_{\mathrm{CP}}\right)+m_{3}^{2} s_{13}^{4}\right]^{1 / 2}
$$

where $m_{2}, m_{3}$ are the light neutrino masses, $c_{i j}=\cos \theta_{i j}, s_{i j}=\sin \theta_{i j}$ are the mixing angles, while $\delta_{\mathrm{CP}}$ is Dirac CP phase and $\alpha_{31}, \alpha_{21}$ are the Majorana phases. ${ }^{5}$ Varying the mixing angles and the phases to cover $\cos \left(\alpha_{31}-\alpha_{21}-2 \delta_{\mathrm{CP}}\right)= \pm 1$, we get a range of $m_{\nu}^{e e}$ for the normal hierarchy which lies in between $1 \mathrm{meV}$ and $4.3 \mathrm{meV}$. This range is in complete agreement with the figure 6 which we obtain after satisfying the neutrino oscillation data (as given in section 3) at the time of diagonalising neutrino mass matrix $m_{\nu}$. Magenta points with a real light neutrino mass matrix correspond to $\delta_{\mathrm{CP}}=0$ and $\left(\alpha_{31}-\alpha_{21}\right)=\pi$, so they point towards the lowest possible value of the neutrinoless double beta decay rate, while the small spread in the $Y_{B}-m_{\nu}^{e e}$ plane is mainly due to the variation of $\theta_{12}$ and $m_{2}$ within their experimental ranges. Hence, with a purely imaginary second column of $M_{D}$, we can satisfy both neutrino oscillation data and produce the baryon and DM asymmetry of the Universe with the Dirac CP phase $\delta_{\mathrm{CP}}=0$ and $\epsilon_{l}=\epsilon_{D}$. This is the case of the minimal number of parameters in the neutrino sector.

In the case of a general $M_{D}$ matrix (shown by the blue points), we can obtain higher values of $m_{\nu}^{e e}$ and hence it will be easier for $0 \nu \beta \beta$ experiments to test this scenario. The higher values of $m_{\nu}^{e e} \sim 4.2 \mathrm{meV}$ imply as well a Dirac CP phase $\delta_{\mathrm{CP}}=-\frac{\pi}{2}$ (in accordance with the recent data from the neutrino oscillation experiments) when $\left(\alpha_{31}-\alpha_{21}\right)=\pi$. Therefore, with a generic $M_{D}$ matrix, we can satisfy the recent data of the neutrino oscillation parameters and produce the baryon and DM asymmetry of the Universe also with large Dirac CP phase, $\delta_{\mathrm{CP}} \sim-\frac{\pi}{2}$.

\section{Direct detection of Dark Matter}

In our model, although DM only has a dark $\mathrm{SU}(2)_{D}$ charge, it can still talk to the visible sector through the exchange of SM-like Higgs boson $h_{1}$ and dark sector Higgs $h_{2}$ respectively and the corresponding Feynman diagram is shown in figure 7.

The expression for the spin-independent scattering cross section between DM and nucleon mediated by scalars $h_{1}, h_{2}$ is given by

$$
\sigma_{\mathrm{SI}}=\frac{\mu_{\mathrm{red}}^{2}}{\pi}\left[\frac{M_{N} f_{N}}{v}\left(\frac{g_{\psi_{1} \psi_{1} h_{2}} \sin \zeta}{M_{h_{2}}^{2}}+\frac{g_{\psi_{1} \psi_{1} h_{1}} \cos \zeta}{M_{h_{1}}^{2}}\right)\right]^{2},
$$

where $\mu_{\text {red }}=\frac{M_{N} M_{\mathrm{DM}}}{M_{N}+M_{\mathrm{DM}}}$ is the reduced mass and $f_{N} \sim 0.3$ [88]. The DM couplings with the scalars have the following form

$$
\begin{aligned}
& g_{\psi_{1} \psi_{1} h_{1}}=-\frac{\lambda_{1}}{\sqrt{2}} \sin \zeta=-\frac{m_{\mathrm{DM}}}{v_{D}} \sin \zeta \\
& g_{\psi_{1} \psi_{1} h_{2}}=\frac{\lambda_{1}}{\sqrt{2}} \cos \zeta=\frac{m_{\mathrm{DM}}}{v_{D}} \cos \zeta,
\end{aligned}
$$

\footnotetext{
${ }^{5}$ Note that for one massless neutrino eigenstate, only one Majorana phase, given as the difference between $\alpha_{31}$ and $\alpha_{21}$, is physical.
} 


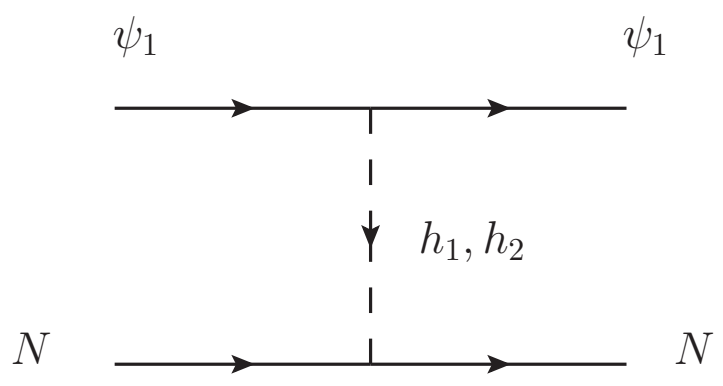

Figure 7. Feynman diagram for the spin-independent scattering cross section of Dark Matter with nucleon mediated by both SM-like Higgs $h_{1}$ and hidden sector Higgs $h_{2}$.

so we see that we have a negative interference and full cancellation for equal masses of the dark and SM Higgs fields, where the mixing in the scalar sector vanishes. Indeed we obtain

$$
\sigma_{\mathrm{SI}}=\frac{\mu_{\mathrm{red}}^{2}}{\pi}\left[\frac{M_{N} f_{N}}{v} \frac{\left|\lambda_{1}\right| \sin 2 \zeta}{2 \sqrt{2} M_{h_{1}}^{2}}\left(1-\frac{M_{h_{1}}^{2}}{M_{h_{2}}^{2}}\right)\right]^{2} .
$$

The masses $M_{h_{1}}, M_{h_{2}}$ and mixing angle $\zeta$ are given in eqs. (2.7), (2.6) while $\lambda_{1}$ is the Yukawa coupling between $\Psi_{1 L}, \tilde{\phi_{D}}$ and $\psi_{1 R}$, related as well to the Dark Matter mass by eq. (2.9).

In figure 8 , we plot the variation of the spin-independent DM-nucleon scattering cross section with the DM mass. In generating the plot, we have kept $\lambda_{1}$ fixed at unity while DM mass has been varied in the range $10 \mathrm{GeV}$ to $10^{4} \mathrm{GeV}$ by appropriately adjusting $v_{D}$. The mixing angle $\zeta$ has been scanned over its present allowed range i.e $\zeta \leq 10^{-1} \mathrm{rad}$. From the figure 7 , we see that a part of the parameter space, corresponding to large $\zeta$ and heavy $M_{h_{2}}$, is already ruled out by the null results at various direct detection experiments like PandaX-II [61] and Xenon1T [60], however enough parameter space is still open and can be tested in the future experiments like Darwin [59].

\section{Conclusion}

In this work we have tried to solve three major puzzles of cosmology by the presence of two $\mathrm{RH}$ neutrinos and a Dark Sector charged under an $\mathrm{SU}(2)_{D}$. The hidden sector of the model is chosen to resemble the SM electroweak sector, but with just two non mixing families, so that the mass of the DM particles could be similar to the SM fermions and the presence of the $\mathrm{SU}(2)_{D}$ interaction is crucial for annihilating away all the symmetric Dark Matter components.

We generate the neutrino mass through the Type-I seesaw mechanism, while both the lepton (later processed into baryons) and a DM asymmetries are produced from the decay of the lightest RH neutrino. Hence, all the three BSM phenomena have a common origin. Moreover, the $\mathrm{CP}$ violation in both sectors is related to complex entries in the neutrino Yukawa couplings. Two RH neutrinos with hierarchical masses are sufficient to accommodate all the present oscillation data and satisfy successfully the present day 


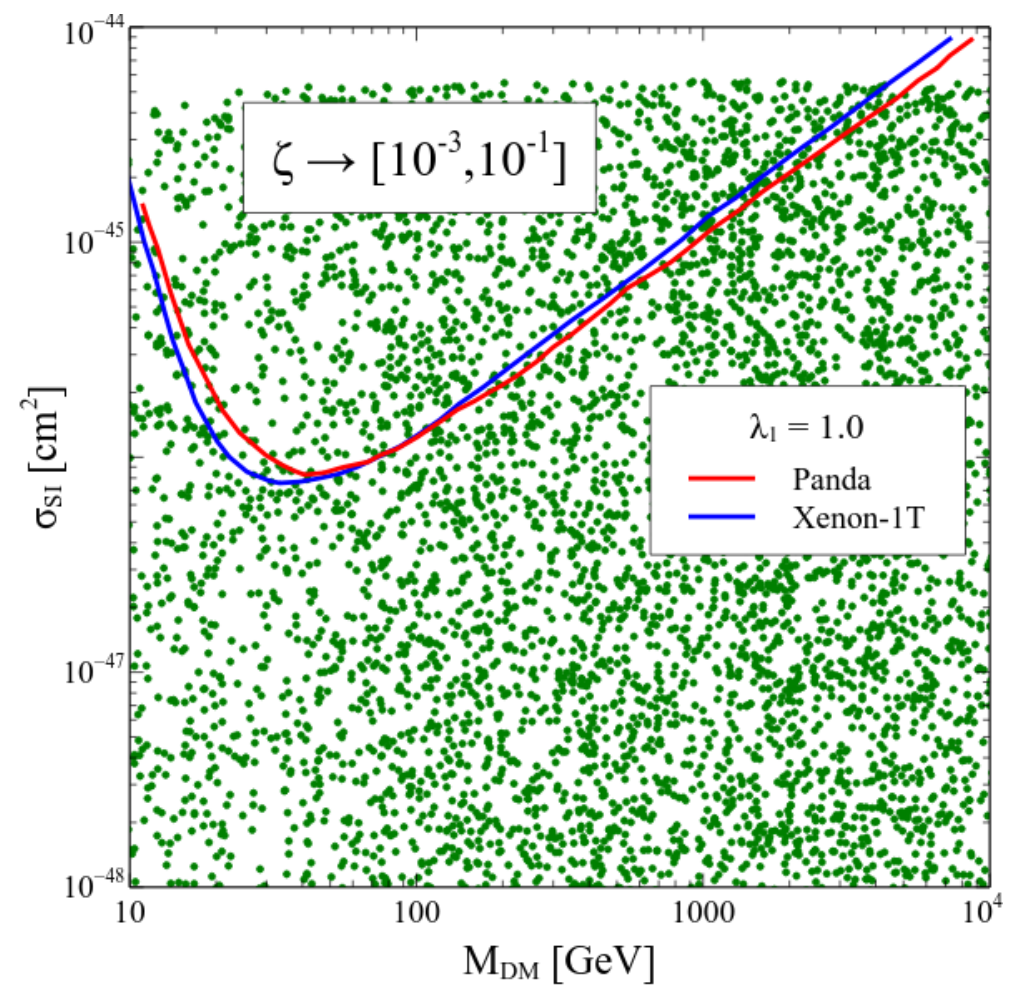

Figure 8. Variation of $\sigma_{\mathrm{SI}}$ with DM mass where the scalar mixing angle $\zeta$ has been varied in the range as shown in legend. Other parameters have been kept fixed $M_{h_{1}}=125.5 \mathrm{GeV}, M_{h_{2}}=1.5 \mathrm{TeV}$ and $\lambda_{1}=1.0$.

bounds on the sum of the light neutrino masses. We have shown that the limited number of parameters in the model results in strong correlations among some of the entries in the Dirac and Majorana mass matrices.

Due to the strong hierarchy, we can generate the baryon asymmetry of the Universe and an asymmetric Dark Matter component at the same time from the decay of the lighter RH neutrino state. Also in this case, two RH neutrino states are enough to give both, as long as they have similar Yukawa coupling with the light neutrinos and the additional dark fermions, so that the two decays can have naturally a similar decay rate and branching fraction. As CP violation is sourced in both sectors by the neutrino Yukawa $y_{i j}$, comparable values of $\epsilon$ for leptons and the Dark Matter are expected in most parameter space. Indeed, the two CP asymmetries in the decays are exactly equal if the neutrino Dirac mass has a purely imaginary column, which gives a real Majorana mass matrix for the light neutrinos and a vanishing Dirac phase. We expect nevertheless to have one non-trivial Majorana phase, that could lead to a partial cancellation in the matrix element for neutrinoless $\beta \beta$ decay. Similarly the CP asymmetry are similar as well in the case $\alpha_{2} \gg \alpha_{1}$ for the Dark sector Yukawa with the RH neutrinos. In other cases we have $\epsilon_{D}<\epsilon_{l}$, but this can be partially compensated by the presence of less effective wash-out processes or in any case by a larger DM mass. For specific choices of the parameters, i.e. the imaginary parts of the Yukawas and Dark Matter mass, we can simultaneously satisfy neutrino oscillation 
parameters bounds and produce the correct value of the baryon asymmetry and of the Dark Matter energy density.

In this scenario the visible and dark sector do not communicate only through the neutrino portal: indeed after the electroweak and dark $\mathrm{SU}(2)_{D}$ symmetries are broken, a mixing appears also in the scalar sector, so that the physical Higgs field contains also a small component of Dark Higgs and can couple to Dark Matter. For light Dark Matter we therefore expect an invisible contribution to the Higgs width proportional to the mixing angle in the scalar sector. If the Dark Matter is heavier than half of the Higgs mass, an observable signal could still appear in the Direct Detection experiments and from the production of Dark Matter at colliders through an off-shell Higgs. Moreover, as in many models with an extended Higgs sector, we can also expect to detect exotic scalars at colliders. Regarding the heavier Higgs state $h_{2}$, Direct Detection and collider experiments are in our model highly complementary, as for heavy $M_{h_{2}}$, whereas the production of $h_{2}$ at colliders is suppressed, the scattering cross section with nucleons becomes larger for fixed mixing angle $\zeta$. Note as well that in this type of model, as in all models with extended Higgs sector, the Higgs self-couplings are modified in a characteristic way and that may be observable even when all the Dark Sector particles are beyond the present collider reach, see e.g. [89, 90].

\section{Acknowledgments}

The authors would like to thank the Department of Atomic Energy (DAE) Neutrino Project under the plan project of Harish-Chandra Research Institute. AB would like to thank the organiser of nu HoRIzons VII (http://www.hri.res.in/ nuhorizons/nuhri7/) for an invitation, where this work was initiated. He also acknowledges SERB, Govt. of India for financial support through NPDF fellowship (PDF/2017/000490) during the early stage of this work. LC would like to thank the Harish-Chandra Research Institute for hospitality during the initial stages of this work. This project has received funding from the European Union's Horizon 2020 research and innovation programme InvisiblesPlus RISE under the Marie Sklodowska-Curie grant agreement No. 690575. This project has received funding from the European Union's Horizon 2020 research and innovation programme Elusives ITN under the Marie Sklodowska-Curie grant agreement No. 674896. 


\section{A Expression for the Majorana mass matrix of light neutrinos}

Here we have given the expression of all the elements of the light neutrino mass matrix $m_{\nu}$ (using eq. (3.1)) in terms of the Yukawa couplings and the RH neutrino masses.

$$
\begin{aligned}
& \left(\tilde{m}_{\nu}\right)_{11}=\frac{y_{e e}^{2}}{M_{N_{1}}}+\frac{\left(y_{e \mu}^{R}\right)^{2}-\left(y_{e \mu}^{I}\right)^{2}}{M_{N_{2}}}-i \frac{2 y_{e \mu}^{R} y_{e \mu}^{I}}{M_{N_{2}}}, \\
& \left(\tilde{m}_{\nu}\right)_{12}=\frac{y_{e e} y_{\mu e}}{M_{N_{1}}}+\frac{y_{e \mu}^{R} y_{\mu \mu}^{R}-y_{e \mu}^{I} y_{\mu \mu}^{I}}{M_{N_{2}}}-i \frac{y_{e \mu}^{R} y_{\mu \mu}^{I}+y_{e \mu}^{I} y_{\mu \mu}^{R}}{M_{N_{2}}}, \\
& \left(\tilde{m}_{\nu}\right)_{13}=\frac{y_{e e} y_{\tau e}}{M_{N_{1}}}+\frac{y_{e \mu}^{R} y_{\tau \mu}^{R}-y_{e \mu}^{I} y_{\tau \mu}^{I}}{M_{N_{2}}}-i \frac{y_{e \mu}^{I} y_{\tau \mu}^{R}+y_{e \mu}^{R} y_{\tau \mu}^{I}}{M_{N_{2}}}, \\
& \left(\tilde{m}_{\nu}\right)_{21}=\left(\tilde{m}_{\nu}\right)_{12} \text {, } \\
& \left(\tilde{m}_{\nu}\right)_{22}=\frac{y_{\mu e}^{2}}{M_{N_{1}}}+\frac{\left(y_{\mu \mu}^{R}\right)^{2}-\left(y_{\mu \mu}^{I}\right)^{2}}{M_{N_{2}}}-i \frac{2 y_{\mu \mu}^{R} y_{\mu \mu}^{I}}{M_{N_{2}}} \\
& \left(\tilde{m}_{\nu}\right)_{23}=\frac{y_{\mu e} y_{\tau e}}{M_{N_{1}}}+\frac{y_{\mu \mu}^{R} y_{\tau \mu}^{R}-y_{\mu \mu}^{I} y_{\tau \mu}^{I}}{M_{N_{2}}}-i \frac{y_{\mu \mu}^{I} y_{\tau \mu}^{R}+y_{\mu \mu}^{R} y_{\tau \mu}^{I}}{M_{N_{2}}}, \\
& \left(\tilde{m}_{\nu}\right)_{31}=\left(\tilde{m}_{\nu}\right)_{13}, \\
& \left(\tilde{m}_{\nu}\right)_{32}=\left(\tilde{m}_{\nu}\right)_{23} \text {, } \\
& \left(\tilde{m}_{\nu}\right)_{33}=\frac{y_{\tau e}^{2}}{M_{N_{1}}}+\frac{\left(y_{\tau \mu}^{R}\right)^{2}-\left(y_{\tau \mu}^{I}\right)^{2}}{M_{N_{2}}}-i \frac{2 y_{\tau \mu}^{R} y_{\tau \mu}^{I}}{M_{N_{2}}}, \\
& m_{\nu}=-\frac{v^{2}}{2}\left(\begin{array}{lll}
\left(\tilde{m}_{\nu}\right)_{11} & \left(\tilde{m}_{\nu}\right)_{12} & \left(\tilde{m}_{\nu}\right)_{13} \\
\left(\tilde{m}_{\nu}\right)_{21} & \left(\tilde{m}_{\nu}\right)_{22} & \left(\tilde{m}_{\nu}\right)_{23} \\
\left(\tilde{m}_{\nu}\right)_{31} & \left(\tilde{m}_{\nu}\right)_{32} & \left(\tilde{m}_{\nu}\right)_{33}
\end{array}\right) .
\end{aligned}
$$

We see from these expressions that if the second column of the Yukawa matrix is imaginary, i.e. for $y_{i \mu}^{R}=0$, the light neutrino mass is the sum of two degenerated real matrices, each with a single non-zero eigenvalue and opposite sign. If the massive eigenvectors of the two matrices are orthogonal to each other, we have then simply two opposite-sign mass eigenstates and one zero mass eigenstate as:

$$
\begin{aligned}
& m_{3}=-\frac{v^{2}}{2} \frac{\sum_{i} y_{i e}^{2}}{M_{1}}, \\
& m_{2}=\frac{v^{2}}{2} \frac{\sum_{i}\left(y_{i \mu}^{I}\right)^{2}}{M_{2}}, \\
& m_{1}=0 .
\end{aligned}
$$

So we have to choose the hierarchy in $M_{i}$ and the Yukawa couplings appropriately in order to match the measured mass differences. If the two massive eigenvectors are not orthogonal, a more complex mixing pattern appears and the two mass eigenstates obtain contributions from both heavy $\mathrm{RH}$ neutrinos, nevertheless for hierarchical masses and not so strongly hierarchical Yukawas, still the heaviest mass $m_{3}$ is mostly determined by the lighter mass $M_{1}$. Generically for real $m_{\nu}$, the mixing matrix is real, so that the Dirac phase is exactly vanishing, but the Majorana phases are not, as they have to be chosen 
to give positive light neutrino masses, i.e. we obtain for the mass eigenstates above the Majorana phases $\alpha_{31}=\pi, \alpha_{21}=0$. We expect also in the generic case with a purely imaginary Yukawa column to have two eigenstates with different Majorana phases, leading to a partial cancellation in the matrix element for neutrinoless double beta decay:

$$
m_{\beta \beta}=\left|\sum_{i} m_{i} U_{e i}^{2}\right|=\left|m_{3} \sin ^{2} \theta_{13}-m_{2} \cos ^{2} \theta_{13} \sin ^{2} \theta_{12}\right| \sim 10^{-2} \mathrm{eV} .
$$

\section{B Expression of $\gamma_{D_{1}}, \gamma_{\phi_{h}, s}^{1}$ and $\gamma_{\phi_{h}, t}^{1}$ :}

Expression of $\gamma_{D_{1}}$ takes the following form [81],

$$
\gamma_{D_{1}}=n_{N_{1}}^{e q} \frac{K_{1}(Z)}{K_{2}(z)} \Gamma_{N_{1}},
$$

where $n_{N_{1}}^{e q}$ is the equilibrium number density of the RH neutrino $N_{1}$ and $K_{n}(z)$ is the $n$th order modified Bessel function of second kind while $\Gamma_{N_{1}}$ is the total decay width of $N_{1}$. The expression of $\Gamma_{N_{1}}$ is given in eq. (4.6).

Further, the general expression of $\gamma(a+b \leftrightarrow i+j+\ldots)$ for a two body scattering process $a+b \leftrightarrow i+j+\ldots$ is given by [81],

$$
\gamma(a+b \leftrightarrow i+j+\ldots)=\frac{T}{64 \pi^{4}} \int_{\left(M_{a}+M_{b}\right)^{2}}^{\infty} d s \hat{\sigma}(s) \sqrt{s} K_{1}\left(\frac{\sqrt{s}}{T}\right),
$$

where, $s$ is one of the Mandelstam variables which physically represents square of the centre of mass energy for a scattering process in centre of momentum frame. Moreover, $\hat{\sigma}(s)$ is the reduced cross section for the scattering process $a+b \leftrightarrow i+j+\ldots$, which is related to the actual cross section by the following relation

$$
\hat{\sigma}(s)=\frac{8}{s}\left[\left(p_{a} \cdot p_{b}\right)^{2}-M_{a}^{2} M_{b}^{2}\right] \sigma(s) .
$$

Here, $p_{i}$ and $M_{i}$ are the three momentum and mass of the species $i$ respectively. The expressions of reduced cross sections for the processes $N_{1}+l \rightarrow \bar{t}+q$ (s-channel process mediated by $\left.\phi_{h}\right)$ and $N_{1}+t \rightarrow \bar{l}+q\left(t\right.$-channel process mediated by $\left.\phi_{h}\right)$ are given as

$$
\begin{aligned}
& \hat{\sigma}_{\phi_{h}, s}=\frac{3 \pi \alpha^{2} M_{t}^{2}}{M_{W}^{4} \sin ^{4} \theta_{w}}\left(M_{D}^{\dagger} M_{D}\right)_{11}\left[\frac{s-M_{N_{1}}^{2}}{s}\right]^{2}, \\
& \hat{\sigma}_{\phi_{h}, t}=\frac{3 \pi \alpha^{2} M_{t}^{2}}{M_{W}^{4} \sin ^{4} \theta_{w}}\left(M_{D}^{\dagger} M_{D}\right)_{11}\left[\frac{s-M_{N_{1}}^{2}}{s}+\frac{M_{N_{1}}^{2}}{s} \ln \left(\frac{s-M_{N_{1}}^{2}+M_{h_{1}}^{2}}{M_{h_{1}}^{2}}\right)\right],
\end{aligned}
$$

where $\alpha=\frac{g^{2} \sin ^{2} \theta_{w}}{4 \pi}, g$ being the $\mathrm{SU}(2)_{L}$ gauge coupling and $\theta_{w}$ is the weak mixing angle (Weinberg angle).

Open Access. This article is distributed under the terms of the Creative Commons Attribution License (CC-BY 4.0), which permits any use, distribution and reproduction in any medium, provided the original author(s) and source are credited. 


\section{References}

[1] C.L. Cowan, F. Reines, F.B. Harrison, H.W. Kruse and A.D. McGuire, Detection of the free neutrino: A Confirmation, Science 124 (1956) 103 [INSPIRE].

[2] Super-Kamiokande collaboration, Evidence for oscillation of atmospheric neutrinos, Phys. Rev. Lett. 81 (1998) 1562 [hep-ex/9807003] [INSPIRE].

[3] SNO collaboration, Direct evidence for neutrino flavor transformation from neutral current interactions in the Sudbury Neutrino Observatory, Phys. Rev. Lett. 89 (2002) 011301 [nucl-ex/0204008] [INSPIRE].

[4] KAMLAND collaboration, First results from KamLAND: Evidence for reactor anti-neutrino disappearance, Phys. Rev. Lett. 90 (2003) 021802 [hep-ex/0212021] [INSPIRE].

[5] DAyA BAy collaboration, Measurement of the Reactor Antineutrino Flux and Spectrum at Daya Bay, Phys. Rev. Lett. 116 (2016) 061801 [Erratum ibid. 118 (2017) 099902] [arXiv: 1508.04233] [INSPIRE].

[6] RENO collaboration, Observation of Energy and Baseline Dependent Reactor Antineutrino Disappearance in the RENO Experiment, Phys. Rev. Lett. 116 (2016) 211801 [arXiv: 1511.05849] [INSPIRE].

[7] Double CHOOZ collaboration, Improved measurements of the neutrino mixing angle $\theta_{13}$ with the Double CHOOZ detector, JHEP 10 (2014) 086 [Erratum ibid. 02 (2015) 074] [arXiv: 1406.7763] [INSPIRE].

[8] T2K collaboration, Measurements of neutrino oscillation in appearance and disappearance channels by the T2K experiment with $6.6 \times 10^{20}$ protons on target, Phys. Rev. D 91 (2015) 072010 [arXiv: 1502.01550] [INSPIRE].

[9] T2K collaboration, Anti-neutrino oscillations with T2K, arXiv:1508.06153 [INSPIRE].

[10] NOvA collaboration, First measurement of electron neutrino appearance in NOvA, Phys. Rev. Lett. 116 (2016) 151806 [arXiv:1601.05022] [INSPIRE].

[11] NOvA collaboration, First measurement of muon-neutrino disappearance in NOvA, Phys. Rev. D 93 (2016) 051104 [arXiv:1601.05037] [INSPIRE].

[12] Y. Sofue and V. Rubin, Rotation curves of spiral galaxies, Ann. Rev. Astron. Astrophys. 39 (2001) 137 [astro-ph/0010594] [INSPIRE].

[13] D. Clowe, A. Gonzalez and M. Markevitch, Weak lensing mass reconstruction of the interacting cluster 1E0657-558: Direct evidence for the existence of dark matter, Astrophys. J. 604 (2004) 596 [astro-ph/0312273] [INSPIRE].

[14] D. Harvey, R. Massey, T. Kitching, A. Taylor and E. Tittley, The non-gravitational interactions of dark matter in colliding galaxy clusters, Science 347 (2015) 1462 [arXiv: 1503.07675] [INSPIRE].

[15] M. Bartelmann and P. Schneider, Weak gravitational lensing, Phys. Rept. 340 (2001) 291 [astro-ph/9912508] [INSPIRE].

[16] WMAP collaboration, Nine-Year Wilkinson Microwave Anisotropy Probe (WMAP) Observations: Cosmological Parameter Results, Astrophys. J. Suppl. 208 (2013) 19 [arXiv: 1212.5226] [INSPIRE].

[17] Planck collaboration, Planck 2015 results. XIII. Cosmological parameters, Astron. Astrophys. 594 (2016) A13 [arXiv:1502.01589] [INSPIRE]. 
[18] Planck collaboration, Planck 2018 results. VI. Cosmological parameters, arXiv: 1807.06209 [INSPIRE].

[19] M. Taoso, G. Bertone and A. Masiero, Dark Matter Candidates: A Ten-Point Test, JCAP 03 (2008) 022 [arXiv: 0711.4996] [INSPIRE].

[20] S. Roy Choudhury and S. Choubey, Updated Bounds on Sum of Neutrino Masses in Various Cosmological Scenarios, JCAP 09 (2018) 017 [arXiv: 1806.10832] [INSPIRE].

[21] A.D. Sakharov, Violation of CP Invariance, $C$ asymmetry and baryon asymmetry of the universe, Pisma Zh. Eksp. Teor. Fiz. 5 (1967) 32 [InSPIRE].

[22] J. Schechter and J.W.F. Valle, Neutrino Masses in $\mathrm{SU}(2) \times \mathrm{U}(1)$ Theories, Phys. Rev. D 22 (1980) 2227 [INSPIRE].

[23] R.N. Mohapatra and G. Senjanović, Neutrino Mass and Spontaneous Parity Nonconservation, Phys. Rev. Lett. 44 (1980) 912 [INSPIRE].

[24] M. Fukugita and T. Yanagida, Baryogenesis Without Grand Unification, Phys. Lett. B 174 (1986) 45 [INSPIRE].

[25] L. Covi, E. Roulet and F. Vissani, CP violating decays in leptogenesis scenarios, Phys. Lett. B 384 (1996) 169 [hep-ph/9605319] [INSPIRE].

[26] V.A. Kuzmin, V.A. Rubakov and M.E. Shaposhnikov, On the Anomalous Electroweak Baryon Number Nonconservation in the Early Universe, Phys. Lett. 155B (1985) 36 [INSPIRE].

[27] H. An, S.-L. Chen, R.N. Mohapatra and Y. Zhang, Leptogenesis as a Common Origin for Matter and Dark Matter, JHEP 03 (2010) 124 [arXiv:0911.4463] [INSPIRE].

[28] B. Dutta and J. Kumar, Asymmetric Dark Matter from Hidden Sector Baryogenesis, Phys. Lett. B 699 (2011) 364 [arXiv:1012.1341] [INSPIRE].

[29] A. Falkowski, J.T. Ruderman and T. Volansky, Asymmetric Dark Matter from Leptogenesis, JHEP 05 (2011) 106 [arXiv:1101.4936] [INSPIRE].

[30] M.L. Graesser, I.M. Shoemaker and L. Vecchi, Asymmetric WIMP dark matter, JHEP 10 (2011) 110 [arXiv:1103.2771] [INSPIRE].

[31] S.D. McDermott, H.-B. Yu and K.M. Zurek, Constraints on Scalar Asymmetric Dark Matter from Black Hole Formation in Neutron Stars, Phys. Rev. D 85 (2012) 023519 [arXiv:1103.5472] [INSPIRE].

[32] H. Iminniyaz, M. Drees and X. Chen, Relic Abundance of Asymmetric Dark Matter, JCAP 07 (2011) 003 [arXiv: 1104.5548] [INSPIRE].

[33] C. Kouvaris and P. Tinyakov, Excluding Light Asymmetric Bosonic Dark Matter, Phys. Rev. Lett. 107 (2011) 091301 [arXiv: 1104.0382] [INSPIRE].

[34] C. Arina and N. Sahu, Asymmetric Inelastic Inert Doublet Dark Matter from Triplet Scalar Leptogenesis, Nucl. Phys. B 854 (2012) 666 [arXiv:1108.3967] [INSPIRE].

[35] M.R. Buckley and S. Profumo, Regenerating a Symmetry in Asymmetric Dark Matter, Phys. Rev. Lett. 108 (2012) 011301 [arXiv:1109.2164] [INSPIRE].

[36] T. Lin, H.-B. Yu and K.M. Zurek, On Symmetric and Asymmetric Light Dark Matter, Phys. Rev. D 85 (2012) 063503 [arXiv: 1111.0293] [INSPIRE].

[37] K. Blum, A. Efrati, Y. Grossman, Y. Nir and A. Riotto, Asymmetric Higgsino Dark Matter, Phys. Rev. Lett. 109 (2012) 051302 [arXiv:1201.2699] [INSPIRE]. 
[38] M. Blennow, E. Fernandez-Martinez, O. Mena, J. Redondo and P. Serra, Asymmetric Dark Matter and Dark Radiation, JCAP 07 (2012) 022 [arXiv:1203.5803] [INSPIRE].

[39] N. Okada and O. Seto, Originally Asymmetric Dark Matter, Phys. Rev. D 86 (2012) 063525 [arXiv: 1205.2844] [INSPIRE].

[40] P. Fileviez Perez and M.B. Wise, Baryon Asymmetry and Dark Matter Through the Vector-Like Portal, JHEP 05 (2013) 094 [arXiv: 1303.1452] [INSPIRE].

[41] K. Petraki and R.R. Volkas, Review of asymmetric dark matter, Int. J. Mod. Phys. A 28 (2013) 1330028 [arXiv: 1305.4939] [INSPIRE].

[42] B. Bhattacherjee, S. Matsumoto, S. Mukhopadhyay and M.M. Nojiri, Phenomenology of light fermionic asymmetric dark matter, JHEP 10 (2013) 032 [arXiv:1306.5878] [INSPIRE].

[43] K.M. Zurek, Asymmetric Dark Matter: Theories, Signatures and Constraints, Phys. Rept. 537 (2014) 91 [arXiv: 1308.0338] [INSPIRE].

[44] Y. Zhao and K.M. Zurek, Indirect Detection Signatures for the Origin of Asymmetric Dark Matter, JHEP 07 (2014) 017 [arXiv: 1401.7664] [INSPIRE].

[45] F. Bishara and J. Zupan, Continuous Flavor Symmetries and the Stability of Asymmetric Dark Matter, JHEP 01 (2015) 089 [arXiv:1408.3852] [INSPIRE].

[46] A. Hamze, C. Kilic, J. Koeller, C. Trendafilova and J.-H. Yu, Lepton-Flavored Asymmetric Dark Matter and Interference in Direct Detection, Phys. Rev. D 91 (2015) 035009 [arXiv:1410.3030] [INSPIRE].

[47] A. Ibarra, S. Lopez-Gehler, E. Molinaro and M. Pato, Gamma-ray triangles: a possible signature of asymmetric dark matter in indirect searches, Phys. Rev. D 94 (2016) 103003 [arXiv: 1604.01899] [INSPIRE].

[48] N. Narendra, S. Patra, N. Sahu and S. Shil, Baryogenesis via Leptogenesis from Asymmetric Dark Matter and radiatively generated Neutrino mass, Phys. Rev. D 98 (2018) 095016 [arXiv: 1805.04860] [INSPIRE].

[49] P. Van Dong, D.T. Huong, D.A. Camargo, F.S. Queiroz and J.W.F. Valle, Asymmetric Dark Matter, Inflation and Leptogenesis from B - L Symmetry Breaking, Phys. Rev. D 99 (2019) 055040 [arXiv: 1805. 08251] [INSPIRE].

[50] M. Ibe, A. Kamada, S. Kobayashi and W. Nakano, Composite Asymmetric Dark Matter with a Dark Photon Portal, JHEP 11 (2018) 203 [arXiv:1805. 06876] [INSPIRE].

[51] C. Dessert, C. Kilic, C. Trendafilova and Y. Tsai, Addressing Astrophysical and Cosmological Problems With Secretly Asymmetric Dark Matter, arXiv:1811.05534 [INSPIRE].

[52] M. Ibe, A. Kamada, S. Kobayashi, T. Kuwahara and W. Nakano, Ultraviolet Completion of a Composite Asymmetric Dark Matter Model with a Dark Photon Portal, JHEP 03 (2019) 173 [arXiv: 1811.10232] [INSPIRE].

[53] A. Dutta Banik, D. Majumdar and A. Biswas, Possible explanation of indirect gamma ray signatures from hidden sector fermionic dark matter, Eur. Phys. J. C 76 (2016) 346 [arXiv: 1506. 05665] [INSPIRE].

[54] E. Witten, An SU(2) Anomaly, Phys. Lett. B 117 (1982) 324 [INSPIRE].

[55] LUX collaboration, Improved Limits on Scattering of Weakly Interacting Massive Particles from Reanalysis of 2013 LUX Data, Phys. Rev. Lett. 116 (2016) 161301 [arXiv:1512.03506] [INSPIRE]. 
[56] XENON collaboration, Physics reach of the XENON1T dark matter experiment, JCAP 04 (2016) 027 [arXiv: 1512.07501] [INSPIRE].

[57] SuperCDMS collaboration, Search for Low-Mass Weakly Interacting Massive Particles with SuperCDMS, Phys. Rev. Lett. 112 (2014) 241302 [arXiv:1402.7137] [InSPIRE].

[58] A. Manalaysay, Dark-matter results from 332 new live days of LUX data, http://lux.brown.edu/talks/IDM2016_332LiveDaysLUX.pdf.

[59] DARWIN collaboration, DARWIN: towards the ultimate dark matter detector, JCAP 11 (2016) 017 [arXiv: 1606.07001] [INSPIRE].

[60] XENON collaboration, First Dark Matter Search Results from the XENON1T Experiment, Phys. Rev. Lett. 119 (2017) 181301 [arXiv:1705.06655] [INSPIRE].

[61] PandaX-II collaboration, Dark Matter Results From 54-Ton-Day Exposure of PandaX-II Experiment, Phys. Rev. Lett. 119 (2017) 181302 [arXiv:1708.06917] [INSPIRE].

[62] A. Falkowski, J. Juknevich and J. Shelton, Dark Matter Through the Neutrino Portal, arXiv:0908.1790 [INSPIRE].

[63] V. González-Macías, J.I. Illana and J. Wudka, A realistic model for Dark Matter interactions in the neutrino portal paradigm, JHEP 05 (2016) 171 [arXiv:1601.05051] [INSPIRE].

[64] M. Escudero, N. Rius and V. Sanz, Sterile neutrino portal to Dark Matter I: The U(1) $B-L$ case, JHEP 02 (2017) 045 [arXiv: 1606.01258] [INSPIRE].

[65] M. Escudero, N. Rius and V. Sanz, Sterile Neutrino portal to Dark Matter II: Exact Dark symmetry, Eur. Phys. J. C 77 (2017) 397 [arXiv:1607.02373] [INSPIRE].

[66] B. Batell, T. Han, D. McKeen and B. Shams Es Haghi, Thermal Dark Matter Through the Dirac Neutrino Portal, Phys. Rev. D 97 (2018) 075016 [arXiv:1709.07001] [INSPIRE].

[67] M. Chianese and S.F. King, The Dark Side of the Littlest Seesaw: freeze-in, the two right-handed neutrino portal and leptogenesis-friendly fimpzillas, JCAP 09 (2018) 027 [arXiv: 1806.10606] [INSPIRE].

[68] ATLAS and CMS collaborations, Measurements of the Higgs boson production and decay rates and constraints on its couplings from a combined ATLAS and CMS analysis of the LHC pp collision data at $\sqrt{s}=7$ and $8 \mathrm{TeV}$, JHEP 08 (2016) 045 [arXiv:1606.02266] [INSPIRE].

[69] ATLAS collaboration, Combined measurements of Higgs boson production and decay in the $H \rightarrow Z Z^{*} \rightarrow 4 \ell$ and $H \rightarrow \gamma \gamma$ channels using $\sqrt{s}=13$ TeV pp collision data collected with the ATLAS experiment, ATLAS-CONF-2017-047.

[70] CMS collaboration, Combined measurements of the Higgs boson's couplings at $\sqrt{s}=13$ TeV, CMS-PAS-HIG-17-031.

[71] F. Capozzi, E. Lisi, A. Marrone, D. Montanino and A. Palazzo, Neutrino masses and mixings: Status of known and unknown 3v parameters, Nucl. Phys. B 908 (2016) 218 [arXiv: 1601.07777] [INSPIRE].

[72] T2K collaboration, Indication of Electron Neutrino Appearance from an Accelerator-produced Off-axis Muon Neutrino Beam, Phys. Rev. Lett. 107 (2011) 041801 [arXiv: 1106.2822] [INSPIRE].

[73] Hyper-Kamiokande Proto-Collaboration collaboration, Physics potential of a long-baseline neutrino oscillation experiment using a J-PARC neutrino beam and Hyper-Kamiokande, PTEP 2015 (2015) 053C02 [arXiv:1502.05199] [INSPIRE]. 
[74] DUNE collaboration, Long-Baseline Neutrino Facility (LBNF) and Deep Underground Neutrino Experiment (DUNE): Conceptual Design Report, Volume 2: The Physics Program for DUNE at LBNF, arXiv:1512.06148 [INSPIRE].

[75] DUNE collaboration, Long-Baseline Neutrino Facility (LBNF) and Deep Underground Neutrino Experiment (DUNE): Conceptual Design Report, Volume 4 The DUNE Detectors at LBNF, arXiv:1601.02984 [INSPIRE].

[76] DUNE collaboration, Long-Baseline Neutrino Facility (LBNF) and Deep Underground Neutrino Experiment (DUNE): Conceptual Design Report, Volume 3: Long-Baseline Neutrino Facility for DUNE June 24, 2015, arXiv:1601.05823 [INSPIRE].

[77] DUNE collaboration, Long-Baseline Neutrino Facility (LBNF) and Deep Underground Neutrino Experiment (DUNE): Conceptual Design Report, Volume 1: The LBNF and DUNE Projects, arXiv: 1601.05471 [INSPIRE].

[78] ICAL collaboration, Physics Potential of the ICAL detector at the India-based Neutrino Observatory (INO), Pramana 88 (2017) 79 [arXiv:1505.07380] [INSPIRE].

[79] T2K collaboration, Search for CP-violation in Neutrino and Antineutrino Oscillations by the T2K Experiment with $2.2 \times 10^{21}$ Protons on Target, Phys. Rev. Lett. 121 (2018) 171802 [arXiv: 1807.07891] [INSPIRE].

[80] NOvA collaboration, New constraints on oscillation parameters from $\nu_{e}$ appearance and $\nu_{\mu}$ disappearance in the NOvA experiment, Phys. Rev. D 98 (2018) 032012 [arXiv:1806.00096] [INSPIRE].

[81] M. Plümacher, Baryogenesis and lepton number violation, Z. Phys. C 74 (1997) 549 [hep-ph/9604229] [INSPIRE].

[82] S. Iso, N. Okada and Y. Orikasa, Resonant Leptogenesis in the Minimal B-L Extended Standard Model at TeV, Phys. Rev. D 83 (2011) 093011 [arXiv:1011.4769] [INSPIRE].

[83] S.Y. Khlebnikov and M.E. Shaposhnikov, The Statistical Theory of Anomalous Fermion Number Nonconservation, Nucl. Phys. B 308 (1988) 885 [inSPIRE].

[84] J. Edsjo and P. Gondolo, Neutralino relic density including coannihilations, Phys. Rev. D 56 (1997) 1879 [hep-ph/9704361] [INSPIRE].

[85] N. Arkani-Hamed, A. Delgado and G.F. Giudice, The Well-tempered neutralino, Nucl. Phys. B 741 (2006) 108 [hep-ph/0601041] [INSPIRE].

[86] I. Baldes and K. Petraki, Asymmetric thermal-relic dark matter: Sommerfeld-enhanced freeze-out, annihilation signals and unitarity bounds, JCAP 09 (2017) 028 [arXiv: 1703.00478] [INSPIRE].

[87] F. D'Eramo and J. Thaler, Semi-annihilation of Dark Matter, JHEP 06 (2010) 109 [arXiv: 1003.5912] [INSPIRE].

[88] J.M. Cline, K. Kainulainen, P. Scott and C. Weniger, Update on scalar singlet dark matter, Phys. Rev. D 88 (2013) 055025 [Erratum ibid. D 92 (2015) 039906] [arXiv:1306.4710] [INSPIRE].

[89] D. López-Val, T. Plehn and M. Rauch, Measuring extended Higgs sectors as a consistent free couplings model, JHEP 10 (2013) 134 [arXiv:1308.1979] [INSPIRE].

[90] I.P. Ivanov, Building and testing models with extended Higgs sectors, Prog. Part. Nucl. Phys. 95 (2017) 160 [arXiv: 1702.03776] [INSPIRE]. 